\title{
How Observations of Circumstellar Disk Asymmetries Can Reveal Hidden Planets: Pericenter Glow and its Application to the HR 4796 Disk
}

\author{
M. C. Wyatt, S. F. Dermott, C. M. Telesco, R. S. Fisher, \\ K. Grogan, E. K. Holmes and R. K. Piña \\ Department of Astronomy, University of Florida, Gainesville, FL 32611
}

\begin{abstract}
Recent images of the disks of dust around the young stars HR 4796A (Jayawardhana et al. 1998; Koerner et al. 1998; Schneider et al. 1999; Telesco et al. 1999) and Fomalhaut Holland et al. 1998) show, in each case, a double-lobed feature that may be asymmetric (one lobe may be brighter than the other). A symmetric double-lobed structure is that expected from a disk of dust with a central hole that is observed nearly edge-on (i.e., close to the plane of the disk). This paper shows how the gravitational influence of a second body in the system with an eccentric orbit would cause a brightness asymmetry in such a disk by imposing a "forced eccentricity" on the orbits of the constituent dust particles, thus shifting the center of symmetry of the disk away from the star and causing the dust near the forced pericenter of the perturbed disk to glow. Dynamic modeling of the HR 4796 disk shows that its $\sim 5 \%$ brightness asymmetry could be the result of a forced eccentricity as small as 0.02 imposed on the disk by either the binary companion HR 4796B, or by an unseen planet close to the inner edge of the disk. Since it is likely that a forced eccentricity of 0.01 or higher would be imposed on a disk in a system in which there are planets, but no binary companion, the corresponding asymmetry in the disk's structure could serve as a sensitive indicator of these planets that might otherwise remain undetected.
\end{abstract}

Subject headings: binaries: visual — celestial mechanics, stellar dynamics circumstellar matter — planetary systems — stars: individual (HR 4796)

\section{Introduction}

A new generation of astronomical instrumentations is now making it possible to image the thermal emission from the disks of dust that surround some stars. The disks that have been imaged around main sequence stars (i.e., the Vega-type, or debris, disks) have aroused considerable interest because it is hoped that interpretation of their structure will provide valuable information about the evolution of planetary systems, possibly even leading to the indirect detection of planets hiding in the disks. One such disk is that around the A0V star HR 4796A. Mid-IR $(10-20 \mu \mathrm{m})$ images of the HR 4796 disk (Jayawardhana et al. 1998; Koerner et al. 1998; Telesco et al. 1999) 
show that its emission is concentrated in two lobes, one on either side of the star, indicating that the disk is being observed nearly edge-on, and that its inner region is almost completely devoid of dust. The same double-lobed feature is seen in NICMOS $(1.1 \mu \mathrm{m})$ images of the disk's scattered light (Schneider et al. 1999). The size of the disk's central cavity, which was previously inferred from the star's spectral energy distribution (Jura et al. 1993), is approximately 40 AU in radius from the star, about the same size as the solar planetary system. Since the age of the HR 4796 system, $t_{\text {sys }} \approx 10$ Myr (Stauffer, Hartmann, \& Barrado Y Navascués 1995; Jura et al. 1998) places it at a stage in its evolution when the formation of any planets is expected to be almost complete (e.g., Lissauer 1993), many authors have speculated that the central cavity could be indicative of planetary formation in this inner region (Jura et al. 1995; Jayawardhana et al. 1998; Koerner et al. 1998; Jura et al. 1998). A similar double-lobed feature has been observed in the disk around the star Fomalhaut, with a similar explanation proposed for its origin (Holland et al. 1998). Some authors, however, remain skeptical about the existence of planets in these systems (e.g., Kalas 1998.).

Observations of the HR 4796 disk show a further interesting feature: the disk's lobes appear to be of unequal brightness, although this observed asymmetry is of low statistical significance $(\sim 1.8 \sigma)$. Mid-IR observations of the disk are described in a companion paper by Telesco et al. (1999, hereafter T99), and their IHW18 $(18.2 \mu \mathrm{m})$ waveband observation (their Fig. 4b) is reproduced in this paper in Fig. Ba. Their observation suggests that the NE lobe (on the left of the image in Fig. 8a) is $\sim 5 \%$ brighter than the SW lobe. This lobe asymmetry may also be apparent in the NICMOS $(1.1 \mu \mathrm{m})$ images of the disk (Schneider et al. 1999), and in the $\sim 20$ $\mu \mathrm{m}$ images of Koerner et al. (1998). The brightnesses of the Fomalhaut disk's lobes also appear to be asymmetric (Holland et al. 1998). There are many possible explanations for the HR 4796 disk's lobe asymmetry. This paper describes a model of the T99 observation that provides one possible dynamical explanation for the asymmetry: that it is the long-term consequence of the gravitational perturbations of one or more massive bodies on the disk (i.e., the consequence of the system's "secular perturbations").

Quite apart from any speculation about a nascent planetary system in the inner region of the HR 4796 disk, we know that the disk must have been gravitationally perturbed, since HR 4796 is a visual binary system. The M dwarf star HR 4796B, located at a projected distance of $517 \mathrm{AU}$ (Jura et al. 1998), is the common proper motion companion of HR 4796A (Jura et al. 1993). An understanding of how asymmetries in the observed structure of the zodiacal cloud, the tenuous disk of dust in the solar system, are linked to the solar system's planets (Dermott et al. 1999), shows that if there is at least one massive perturber in the HR 4796 system that is on an eccentric orbit, then the system's secular perturbations would have caused the disk's center of symmetry to be offset from the star (Dermott et al. 1998). This offset would mean that the material in one of the disk's observed lobes is closer to the star than that in the other lobe; consequently this lobe would be hotter and brighter. The aim of this paper is to ascertain how large the perturbations would have to be to cause the observed $5 \%$ asymmetry, and to discuss whether perturbations of 
this magnitude are physically realistic, or even to be expected, in this system.

Since this paper is based on the interpretation of a circumstellar disk observation, it starts

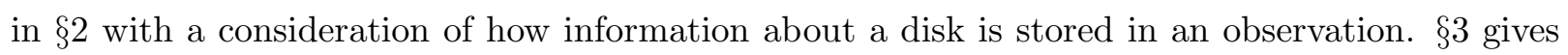
a comprehensive discussion of the physical processes that govern the dynamical evolution of a disk's particles, and sets up a broad theoretical framework (the "dynamic disk") with which to investigate a disk's structure. $\oint$ 团 then shows how secular perturbations cause a disk's structure to have offset and warp asymmetries, and provides evidence that these asymmetries have been observed in the structure of the zodiacal cloud. $\S 5$ uses the theoretical discussions of $\S \S 2$ t 4 to create an offset model for the HR 4796 disk that matches the $18.2 \mu \mathrm{m}$ brightness distribution observed by T99. The interpretation of this model is discussed in $\$ 6$.

\section{The Observable Circumstellar Disk}

The observed brightness of a circumstellar disk comes from two sources: starlight that has been absorbed by the disk particles and re-emitted as thermal radiation (primarily at mid-IR, far-IR, and submillimeter wavelengths at $\lambda>5 \mu \mathrm{m}$ ), and starlight that has been scattered by the disk particles (primarily at optical and near-IR wavelengths at $\lambda<2 \mu \mathrm{m}$ ). This paper only discusses a disk's thermal emission.

\subsection{Thermal Emission of a Single Disk Particle}

A particle of diameter $D$, that is at a distance $r$ from a star, is heated by the stellar radiation to a temperature $T$ that can be calculated from the equilibrium between the energy that the particle absorbs and that which it re-emits as thermal radiation. This temperature depends on the particle's optical properties (Gustafson 1994):

$$
T(D, r)=\left[\left\langle Q_{a b s}\right\rangle_{T_{\star}} /\left\langle Q_{a b s}\right\rangle_{T(D, r)}\right]^{1 / 4} * T_{b b},
$$

where this equation must be solved iteratively, since the particle's temperature appears on both sides of the equation, $\left\langle Q_{a b s}\right\rangle_{T_{\star}}$ and $\left\langle Q_{a b s}\right\rangle_{T(D, r)}$ are the particle's absorption efficiency averaged over the stellar spectrum (which can be approximated as that of a black body radiating at the star's effective temperature $T_{\star}$ ) and the spectrum of a black body radiating at a temperature $T$, and $T_{b b}$ is the equilibrium temperature of the particle if it were a black body:

$$
T_{b b}=278.3 \sqrt{a_{\oplus} / r} *(4 \sigma / A)\left(L_{\star} / L_{\odot}\right)^{1 / 4},
$$

where $T_{b b}$ is given in $\mathrm{K}, \sigma / A$ is the ratio of the particle's cross-sectional area to its surface area (e.g., spherical particles have $\sigma=\pi D^{2} / 4$ and $A=\pi D^{2}$, giving $\sigma / A=1 / 4$ ), and $L_{\star}$ and $L_{\odot}$ are the luminosities of the star and the Sun. 
If this particle is at a distance $R_{\oplus}$ from the Earth, the contribution of its thermal emission to the flux density at a wavelength, $\lambda$, received at the Earth is given by:

$$
F_{\nu}(\lambda, D, r)=Q_{a b s}(\lambda, D) B_{\nu}[\lambda, T(D, r)] \Omega(D)
$$

where $B_{\nu}$ is the Planck function, and $\Omega=\sigma / R_{\oplus}^{2}$ is the solid angle subtended at the Earth by the cross-sectional area of the particle.

\subsection{Definition of Disk Structure}

A circumstellar disk consists of particles with a range of sizes, compositions and morphologies. Throughout this paper, however, disk particles are assumed to have the same composition and morphology, and a particle's size is characterized by its diameter, $D$. Disk particles span a range of sizes from $D_{\text {min }}$, the smallest (probably submicron-sized) particles sustainable for a given disk, up to $D_{\max }$, the largest (probably kilometer-sized) members of the disk that were formed from the proto-planetary disk. The spatial distribution of these particles can be defined by $n(D, r, \theta, \phi)$, where $n(D, r, \theta, \phi) d D$ is the volume density (number per unit volume) of particles in the size range $D \pm d D / 2$ at a location in the disk defined by $r$, the radial distance from the star, $\theta$, the longitude relative to an arbitrary direction, and $\phi$, the latitude relative to an arbitrary reference plane. However, since it is a particle's cross-sectional area that is apparent in an observation (eq. [3]), a disk's observable structure is better defined in terms of $\sigma(D, r, \theta, \phi)=n(D, r, \theta, \phi) \sigma$, the cross-sectional area per unit volume per unit diameter.

The definition of a disk's structure can be simplified by assuming the size distribution of its particles to be independent of $\theta$ and $\phi$ :

$$
\sigma(D, r, \theta, \phi)=\bar{\sigma}(D, r) \sigma(r, \theta, \phi)
$$

where $\bar{\sigma}(D, r) d D$ is the proportion of the total cross-sectional area of the disk at $r$ that is in particles in the size range $D \pm d D / 2$, and

$$
\sigma(r, \theta, \phi)=\int_{D_{\min }}^{D_{\max }} \sigma(D, r, \theta, \phi) d D
$$

is the spatial distribution of cross-sectional area of particles of all sizes in the disk.

\subsection{Line of Sight Brightness of a Disk Observation}

To determine the observed brightness of a circumstellar disk in, for example, one pixel of an image of the disk, two components of the observation must be defined: the vector, $\mathbf{R}$, which extends from the observer to the disk, and which describes how the line of sight intersects the disk 
in terms of $r, \theta$, and $\phi$; and the solid angle of the observation, $\Omega_{o b s}$, where $\Omega_{o b s}=d_{p i x}^{2}$ for pixels of width $d_{\text {pix }}$ radians.

Consider a volume element along this line of sight that is at a location in the disk defined by $r, \theta, \phi$, and that has a length $\mathbf{d R}$; the element volume is $d V=\Omega_{o b s} R_{\oplus}^{2} \mathbf{d R}$. The contribution of the thermal emission of the particles in this element to the disk's brightness in the observation is given by:

$$
\begin{aligned}
d F_{\nu}(\lambda, r, \theta, \phi) / \Omega_{o b s} & =\int_{D_{\min }}^{D_{\max }} Q_{a b s}(\lambda, D) B_{\nu}[\lambda, T(D, r)] \sigma(D, r, \theta, \phi) d D \mathbf{d R}, \\
& =P(\lambda, r) \sigma(r, \theta, \phi) \mathbf{d R}
\end{aligned}
$$

where equation (7) uses the simplification for the disk structure given by equation (4), and

$$
P(\lambda, r)=\int_{D_{\min }}^{D_{\max }} Q_{a b s}(\lambda, D) B_{\nu}[\lambda, T(D, r)] \bar{\sigma}(D, r) d D .
$$

Thus, the brightness of this element is not affected by the solid angle of the observation, neither is it affected by the distance of the element from the Earth.

Equation (8) can also be written as:

$$
P(\lambda, r)=\left\langle Q_{a b s}(\lambda, D) B_{\nu}[\lambda, T(D, r)]\right\rangle_{\sigma(D, r)} ;
$$

i.e., $P(\lambda, r)$ is the combination of the particles' optical properties given by $Q_{a b s}(\lambda, D) B_{\nu}[T(D, r), \lambda]$ averaged over the disk's cross-sectional area distribution, $\sigma(D, r)$. Thus, $P(\lambda, r)$ can also be given by:

$$
P(\lambda, r)=Q_{a b s}\left(\lambda, D_{t y p}\right) B_{\nu}\left[\lambda, T\left(D_{t y p}, r\right)\right],
$$

where $D_{t y p}$ is the size of disk particle that characterizes (and hence dominates) the disk's emission. This characteristic particle size could be different in different wavebands, as well as at different distances from the star, but always lies in the range $D_{\min }<D_{\text {typ }}<D_{\max }$; its value can be found by considering the relative contribution of particles of different sizes to $P(\lambda, r)$. However, unless

the optical properties of the particles prevent it, the particles that dominate a disk's emission are also those that contribute most to its cross-sectional area, i.e., they are those that dominate the disk's structure, $\sigma(r, \theta, \phi)$. This is usually the case for mid-IR $(\lambda=10-20 \mu \mathrm{m})$ observations, such as those of T99 (see e.g., \$5.2).

The total brightness of the disk in this observation is the integral of equation (6) over $\mathbf{R}$. Thus, a disk observation is composed of three parts: the disk's structure, the optical properties of the disk particles, and the orientation of the disk to the line of sight.

\subsection{Real Circumstellar Disk Images}

An image of the disk is made up of many pixels, each of which has a different line of sight vector, $\mathbf{R}$, and corresponding brightness. The image at the detector has been convolved with 
the observational point spread function (PSF), a combination of the seeing conditions and the telescope and instrumental optics, which, in the diffraction-limited case, for an ideal instrument, can be approximated as gaussian smoothing with $\mathrm{FWHM}=\lambda / D$, where this $D$ is the diameter of the telescope. The observed image also contains photospheric emission from the point-like star, as well as random noise fluctuations. A useful image of the disk can be recovered by subtracting the image of the star, but the accuracy of this subtraction depends on how well both the PSF and the stellar flux density are known. Further smoothing of the image to increase the signal-to-noise may also prove useful.

\section{The Dynamic Disk}

This section outlines the theoretical framework upon which later discussion of circumstellar disk structure (such as how secular perturbations affect this structure) is based. A disk is a dynamic entity, the constituent particles of which are undergoing constant dynamical and physical evolution; $\$ 3.1$ gives an extensive discussion of the physical processes acting on disk particles. $\$ 3.2$ then summarizes our understanding of the dynamic disk by showing how disk particles can be categorized according to the dominant physical processes affecting their evolution.

\subsection{Physical Processes}

If we are to make generalizations about the physical processes relevant to circumstellar disk evolution, then the zodiacal cloud is the best example of a circumstellar disk on which to base this understanding, since its properties have been determined with a certain degree of confidence. This confidence stems from the wealth of observational, theoretical, and physical evidence describing its present state, its evolutionary history, and the physical environment of the system it is in. The physical processes that are described in this section are those thought to have dominated the evolution of the zodiacal cloud, supposedly since the Sun reached the main sequence (e.g., Leinert \& Grün 1990; Gustafson 1994). These processes should serve as an adequate basis for an understanding of the evolution of the circumstellar disks around other main sequence stars.

There is, however, one obvious distinction between the zodiacal cloud and exosolar dust disks. The emission observed from the zodiacal cloud is dominated by that from dust in the inner solar system (within $5 \mathrm{AU}$ of the Sun), which has its origins in the asteroid belt (Dermott et al. 1984; Grogan et al. 1997) and the short period comets (Sykes et al. 1986). In contrast, the emission observed from exosolar dust disks is dominated by that originating from dust in regions analogous to the Kuiper belt in the solar system, i.e., > 30 AU from the star (e.g., Backman \& Paresce 1993). Relatively little is known about the Kuiper belt, but, like the inner solar system, it appears to be populated with many asteroid- or comet-like objects that are probably the remnants of the solar system's planetary formation phase (e.g., Jewitt 1999). 


\subsubsection{Gravity}

The dominant force acting on all but the smallest disk particles is the gravitational attraction of the star:

$$
F_{\text {grav }}=G M_{\star} m / r^{2},
$$

where $G$ is the gravitational constant, $M_{\star}$ is the mass of the star, $m$ is the mass of the particle, and $r$ is the distance of the particle from the star. All material is assumed to orbit the star on Keplerian (elliptical) orbits, with other forces acting as perturbations to these orbits. The orbit of a particle is defined by the following orbital elements: the semimajor axis, $a$, and eccentricity, $e$, that define the radial extent and shape of the orbit; the inclination, $I$, and longitude of ascending node, $\Omega$, that define the plane of the orbit (relative to an arbitrary reference plane); and the longitude of pericenter, $\tilde{\omega}$, that defines the orientation of the orbit within the orbital plane (relative to an arbitrary reference direction). The orbital period of the particle is given by:

$$
t_{p e r}=\sqrt{\left(a / a_{\oplus}\right)^{3}\left(M_{\odot} / M_{\star}\right)}
$$

where $t_{p e r}$ is given in years, $a_{\oplus}=1 \mathrm{AU}$ is the semimajor axis of the Earth's orbit, and $M_{\odot}$ is the mass of the Sun. At any instant, the location of the particle in its orbit is defined by the true anomaly, $f$, where $f=0^{\circ}$ and $180^{\circ}$ at the pericenter and apocenter, respectively, and its distance from the star, $r$, and its velocity, $v$, are defined by:

$$
\begin{aligned}
r & =a\left(1-e^{2}\right) /(1+e \cos f), \\
v & =\sqrt{G M_{\star}(2 / r-1 / a)} .
\end{aligned}
$$

Since at any given time a particle could be at any point along its orbit, its contribution to the distribution of material in a disk can be described by the elliptical ring that contains the mass of the particle spread out along its orbit, the line density of which varies inversely with the particle's velocity (eq. 14]). Each disk particle has an orbit defined by a different set of orbital elements, with a contribution to the spatial distribution of material in the disk that can be described by a corresponding elliptical ring. Thus, a disk's structure can be defined by the distribution of orbital elements of its constituent particles, $n(D, a, e, I, \Omega, \tilde{\omega})$, where $n(D, a, e, I, \Omega, \tilde{\omega}) d D d a d e d I d \Omega d \tilde{\omega}$ is the number of disk particles with sizes in the range $D \pm d D / 2$, and orbital elements in the range $a \pm d a / 2, e \pm d e / 2, I \pm d I / 2, \Omega \pm d \Omega / 2, \tilde{\omega} \pm d \tilde{\omega} / 2$. A disk's orbital element distribution can be quantified in terms of the evolutionary history of the system and the physical processes acting on the disk's particles. Using techniques such as those described in $\$ 5.1 .2$, the resulting structure can then be compared with the disk's observable structure, $\sigma(D, r, \theta, \phi)$, to link a disk observation with the physics of the particles in that disk. It is often convenient to discuss the dependence of the orbital element distribution on the different parameters separately; e.g., the distribution of semimajor axes, $n(a)$, is defined such that $n(a) d a$ is the total number of particles with orbital semimajor axes in the range $a \pm d a / 2$. 


\subsubsection{Collisions}

A typical disk particle is created by the break-up of a larger "parent" body, either as the result of a collision with another body, or simply by its disintegration. This parent body could have been created by the break-up of an even larger body, and the particle itself will most likely end up as a parent body for particles smaller than itself. This "collisional cascade" spans the complete size range of disk material, and the particles that share a common ancestor are said to constitute a "family" of particles.

The size distribution that results from this collisional cascade can be found from theoretical arguments (Dohnanyi 1969):

$$
n(D) \propto D^{2-3 q}
$$

where $q=11 / 6$; this distribution is expected to hold for disk particles that are large enough not to be affected by radiation forces $(\oint 3.1 .3)$. A disk with this distribution has its mass, $m(D)=n(D) m$, concentrated in its largest particles, while its cross-sectional area, $\sigma(D)=n(D) \sigma$, is concentrated in its smallest particles. Collisions in such a disk are mostly non-catastrophic (see eqs. [A2]-A6]), and a particle in this disk is most likely to be broken up by a particle that has just enough mass (and hence energy) to do so. This in turn means that collisional fragments have velocities, and hence orbital elements, that are almost identical to those of the original particle; i.e., in the absence of other forces, all members of the same family have identical orbits. Due to the interaction of the competing physical processes, the size distribution of disk particles that are affected by radiation forces is only really understood qualitatively ( $\$ \$ 3.1 .3$ and 3.2 .2$)$; their distribution is particularly important, since, in general, a disk's cross-sectional area (and hence its observable structure) is concentrated in these smaller particles.

The importance of collisions in determining a particle's evolution depends on its collisional lifetime, which is discussed in Appendix A. The collisional lifetime of the particles that constitute most of a disk's cross-sectional area (i.e., those that are expected to characterize the disk's mid-IR emission, $\$ 2.3$ ), can be approximated by (eqs. A9] and [A12]):

$$
t_{\text {coll }}\left(D_{\text {typ }}, r\right)=t_{\text {per }}(r) / 4 \pi \tau_{\text {eff }}(r),
$$

where $t_{\text {per }}(r)$ is the average orbital period of particles at $r$ (eq. [12] with $a$ replaced by $r$ ), and $\tau_{\text {eff }}(r)$ is the disk's effective face-on optical depth (eq. A\&]), which would be equal to the disk's true optical depth if its particles had unity extinction efficiency. The collisional lifetime of particles with $D>D_{\text {typ }}$ can be considerably longer than that of equation (16) (see e.g., eq. A15]), and a disk's largest particles, those for which $t_{\text {coll }}(D, r)>t_{\text {sys }}$, may not have suffered any catastrophic collisions since they were first created; such particles are primordial particles. The cascades of very young disks may still contain a significant proportion of primordial particles; i.e., their cascades may not be fully evolved.

The collisional cascade theory is well-supported by evidence from the zodiacal cloud. The size distribution of the largest $(D>3 \mathrm{~km})$ members of the zodiacal cloud's collisional cascade, 
the observable asteroids, is well-approximated by equation (15) (Durda \& Dermott 1997; Durda, Greenberg, \& Jedicke 1998); the distribution of the very largest $(D>30 \mathrm{~km})$ asteroids deviates from this distribution, however, because of the transition from strength-scaling to gravity-scaling for asteroids larger than $\sim 150 \mathrm{~m}$ (Durda et al. 1998). The size distribution of the zodiacal cloud's medium-sized $(1 \mathrm{~mm}<D<3 \mathrm{~km})$ members is also expected to follow equation (15) (Durda \& Dermott 1997), but there is no observational proof of this, since these members are too faint to be seen individually, and too few to be studied collectively (Leinert \& Grün 1990). There is, however, proof that the zodiacal cloud's collisional cascade extends from its largest members down to its smallest dust particles: the shapes of the "dust band" thermal emission features (Low et al. 1984) correspond to those expected from the small $(1-1000 \mu \mathrm{m})$ particles resulting from the break-up, some time ago, of a few very large asteroids, the largest fragments of which are still observable as the asteroids in the Themis, Koronis, and, possibly, Eos families (Dermott et al. 1984; Grogan et al. 1997). The size distribution of the zodiacal cloud's smallest ( $D<1 \mathrm{~mm}$ ) dust particles (e.g., Leinert \& Grün 1990; Love \& Brownlee 1993) can be explained qualitatively (e.g., Grün et al. 1985; see also $\$ 3.1 .3)$.

Analysis of the collision rates of objects in the Kuiper belt (Stern 1995) shows that a collisional cascade should exist here too; there is also evidence to suggest that the Kuiper belt was once more massive than it is today (Jewitt 1999), meaning that in the past collisions would have played a much larger role in determining its structure than they do today, maybe even causing the supposed mass loss (Stern \& Colwell 1997). The size distribution of the observed Kuiper belt objects appears to be slightly steeper than that in the inner solar system $(q>11 / 6$, Jewitt 1999), while observations have been unable, as yet, to determine its dust distribution (Backman, Dasgupta, \& Stencel 1995; Gurnett et al. 1997),

\subsubsection{Radiation Forces, $\beta$}

For most of the collisional cascade, gravity can be considered to be the only significant force acting on disk particles. The smallest particles, however, are significantly affected by their interaction with the photons from the star.

Radiation Pressure Radiation pressure is the component of the radiation force that points radially away from the star. It is inversely proportional to the square of a particle's distance from the star, and is defined for different particles by its ratio to the gravitational force of equation (11) (Gustafson 1994):

$$
\beta(D)=F_{\text {rad }} / F_{\text {grav }}=C_{r}(\sigma / m)\left\langle Q_{p r}\right\rangle_{T_{\star}}\left(L_{\star} / L_{\odot}\right)\left(M_{\odot} / M_{\star}\right)
$$

where $C_{r}=7.65 \times 10^{-4} \mathrm{~kg} / \mathrm{m}^{2}, \sigma / m$ is the ratio of the particle's cross-sectional area to its mass (e.g., $\sigma / m=1.5 / \rho D$ for spherical particles of density $\rho$ ), and $\left\langle Q_{p r}\right\rangle_{T_{\star}}=\int Q_{p r}(D, \lambda) F_{\lambda} d \lambda / \int F_{\lambda} d \lambda$ 
is the particle's radiation pressure efficiency円 averaged over the stellar spectrum, $F_{\lambda}$.

An approximation for large particles is that $\left\langle Q_{p r}\right\rangle_{T_{\star}} \approx 1$; thus, large spherical particles have:

$$
\beta(D) \approx(1150 / \rho D)\left(L_{\star} / L_{\odot}\right)\left(M_{\odot} / M_{\star}\right),
$$

where $\rho$ is measured in $\mathrm{kg} / \mathrm{m}^{3}$, and $D$ in $\mu \mathrm{m}$. This approximation is valid for particles in the solar system with $D>20 \mu \mathrm{m}$ (Gustafson 1994). For particles in exosolar systems, this limit scales with the wavelength at which the star emits most of its energy, $\lambda_{\star} \propto 1 / T_{\star}$; i.e., equation (18) is valid for spherical particles with $D>20\left(T_{\odot} / T_{\star}\right) \mu \mathrm{m}$, where $T_{\odot}=5785 \mathrm{~K}$ is the effective temperature of the Sun. Since $\beta \propto 1 / D$, this means that the smaller a particle, the larger its $\beta$; this holds down to micron-sized particles, smaller than which $\beta$ decreases to a level that is independent of the particle's size (Gustafson 1994).

The effect of radiation pressure is equivalent to reducing the mass of the star by a factor $1-\beta$. This means that a particle for which $\beta \neq 0$ moves slower around the same orbit by a factor of $\sqrt{1-\beta}$ than one for which $\beta=0$ (eq. [14]). It also means that daughter fragments created by the break-up of a parent body move on orbits that can differ substantially from that of the parent. The reason for this, is that while the positions and velocities of a parent and its daughter fragments are the same at the moment of break-up (apart from a small velocity dispersion), their $\beta$ are different, and so the daughter fragments move in effective potentials that are different from that the parent moved in. Daughter fragments created in the break-up of a parent particle that had $\beta=0$, and for which orbital elements at the time of the collision were $a, e, I, \Omega, \tilde{\omega}$, and $f$, move in the same orbital plane as the parent, $I^{\prime}=I$ and $\Omega^{\prime}=\Omega$, but on orbits with semimajor axes, $a^{\prime}$, eccentricities, $e^{\prime}$, and pericenter orientations, $\tilde{\omega}^{\prime}$, that are given by (Burns, Lamy, \& Soter 1979):

$$
\begin{aligned}
a^{\prime} & =a(1-\beta) /\left[1-2 \beta(1+e \cos f) /\left(1-e^{2}\right)\right], \\
e^{\prime} & =(1-\beta)^{-1} \sqrt{e^{2}+2 \beta e \cos f+\beta^{2}} \\
\tilde{\omega}^{\prime}-\tilde{\omega} & =f-f^{\prime}=\arctan [\beta \sin f /(\beta \cos f+e)] .
\end{aligned}
$$

Analysis of equations (19)-(21) shows that the orbits of the largest fragments, those for which $\beta<0.1$, are similar to that of the parent. On the other hand, the smallest fragments, those for which $\beta>0.5\left(1-e^{2}\right) /(1+e \cos f)$, have hyperbolic orbits $\left(e^{\prime}>1\right)$; these particles are known as " $\beta$ meteoroids" 6 . Since $\beta$ meteoroids are lost from the system on the timescale of the orbital period of the parent (eq. [12]), the diameter of particle for which $\beta>0.5$ essentially defines the lower end of the collisional cascade. However, there may also be a population of submicron

\footnotetext{
${ }^{1} \mathrm{~A}$ particle's radiation pressure efficiency is related to its absorption and scattering efficiencies by $Q_{p r}=$ $Q_{a b s}+Q_{s c a}(1-\langle\cos \theta\rangle)$, where $\langle\cos \theta\rangle$ accounts for the asymmetry of the scattered radiation.

${ }^{2}$ Note that particles with $\beta>1$ are $\beta$ meteoroids even if they were not created collisionally, since they "see" a negative mass star.
} 
particles that have $\beta<0.5$ (Gustafson 1994). The intermediate-sized fragments, those for which $0.1<\beta<0.5$, that we call " $\beta$ critical" particles, have orbits that differ substantially from that of the parent. However, the point of closest approach to the star of the orbits of all daughter fragments, irrespective of their size, is the same as that of the parent: combining equations (19) and (20) gives the pericenter distance of daughter fragments, $r_{p}^{\prime}=a^{\prime}\left(1-e^{\prime}\right)$, as

$$
r_{p}^{\prime} / r_{p}=1+e(1-\cos f)+O\left(e^{2}\right) .
$$

The orbits of collisional fragments with different $\beta$ from a parent particle that was on a circular orbit are shown in Fig. 11.

Poynting-Robertson (P-R) Light Drag The component of the radiation force tangential to a particle's orbit is called the P-R drag force. This force is also proportional to $\beta$. It results in an evolutionary decrease in both the semimajor axis and eccentricity of the particle's orbit (Burns et al. 1979):

$$
\begin{aligned}
& \dot{a}_{p r}=-(\alpha / a)\left(2+3 e^{2}\right) /\left(1-e^{2}\right)^{3 / 2}=-2 \alpha / a+O\left(e^{2}\right) \\
& \dot{e}_{p r}=-\left(\alpha / a^{2}\right) 2.5 e /\left(1-e^{2}\right)^{1 / 2}=-2.5 \alpha e / a^{2}+O\left(e^{2}\right),
\end{aligned}
$$

where $\alpha=6.24 \times 10^{-4}\left(M_{\star} / M_{\odot}\right) \beta \mathrm{AU}^{2} /$ year. P-R drag does not change the plane of the particle's orbit, $\dot{I}_{p r}=\dot{\Omega}_{p r}=0$; neither does it affect the orientation of the particle's pericenter, $\dot{\tilde{\omega}}_{p r}=0$. For a particle with zero eccentricity, equation $(23)$ can be solved to find the time it takes for the particle to spiral in from a radial distance of $r_{1}$ to $r_{2}$ :

$$
t_{p r}=400\left(M_{\odot} / M_{\star}\right)\left[\left(r_{1} / a_{\oplus}\right)^{2}-\left(r_{2} / a_{\oplus}\right)^{2}\right] / \beta,
$$

where $t_{p r}$ is given in years.

Consider the daughter fragments created in the break-up of a parent body that was on an orbit at a distance $r$ from the star. The largest fragments are broken up by collisions before their orbits have suffered any significant P-R drag evolution, while the smaller fragments, for which the P-R drag evolution is faster, can reach the star without having encountered another particle (at which point they evaporate). Particles for which P-R drag significantly affects their orbits in their lifetime can be estimated as those for which their collisional lifetime (eq. [16]; the use of this equation is justified in the next paragraph) is longer than their P-R drag lifetime (eq. [25] with $r_{2}=0$ ), i.e., those for which $\beta>\beta_{p r}$, where

$$
\beta_{p r}=5000 \tau_{e f f}(r) \sqrt{\left(M_{\odot} / M_{\star}\right)\left(r / a_{\oplus}\right)} ;
$$

for large spherical particles, this is also those for which $D<D_{p r}$, where

$$
D_{p r}=\left[0.23 / \rho \tau_{e f f}(r)\right]\left(L_{\star} / L_{\odot}\right) \sqrt{\left(M_{\odot} / M_{\star}\right)\left(a_{\oplus} / r\right)},
$$

$\rho$ is measured in $\mathrm{kg} / \mathrm{m}^{3}$, and $D_{p r}$ in $\mu \mathrm{m}$. 
Consider the daughter fragments created in the break-up of an endless supply of parent bodies that are on orbits with the same semimajor axis, $a_{s}$. Ignoring collisional processes, the fragments with orbits that are affected by P-R drag, those with $\beta>\beta_{p r}$, have their semimajor axes distributed from $a=a_{s}$ to $a=0$ according to (eq. [23]):

$$
n(a) \propto 1 / \dot{a}_{p r} \propto a
$$

this corresponds to a volume density distribution that is roughly inversely proportional to distance from the star $[$. If the collisional processes leading to the size distribution of the parent bodies, $n_{s}(D)$, still holds for the production of the P-R drag affected particles, then their size distribution is given by:

$$
n(D) \propto n_{s}(D) / \dot{a}_{p r} \propto n_{s}(D) D .
$$

If $n_{s}(D)$ can be given by equation (15) with $q=11 / 6$, the cross-sectional area of a disk's P-R drag affected particles is concentrated in the largest of these particles, while that of its unaffected particles is concentrated in the smallest of these particles; i.e., most of a disk's cross-sectional area is expected to be concentrated in particles with $D_{t y p} \approx D_{p r}$, justifying the use of equation (16) for the collisional lifetime of these particles.

Observations of the zodiacal cloud at 1 AU show that its effective optical depth here is $\tau_{\text {eff }}=O\left(10^{-7}\right)$. Since these particles originated in the asteroid belt at $\sim 3$ AU, arriving at 1 AU due to the P-R drag evolution of their orbits, the zodiacal cloud's volume density should vary $\propto 1 / r$, and its effective optical depth at $3 \mathrm{AU}$ should be similar to that at $1 \mathrm{AU}$. Assuming zodiacal cloud particles to have a density $\sim 2500 \mathrm{~kg} / \mathrm{m}^{3}$ (Leinert \& Grün 1990), the cross-sectional area of material in the asteroid belt should be concentrated in particles with $D_{p r}=O(500 \mu \mathrm{m})$ (eq. [27]), for which both the collisional lifetime, and the P-R drag lifetime, is $\sim 4$ Myr. The cross-sectional area of material at $1 \mathrm{AU}$ is expected to be concentrated in particles smaller than that in the asteroid belt, since many of the larger particles should have been broken up by collisions before they reach the inner solar system; this is in agreement with observations that show the cross-sectional area distribution at 1 AU to peak for particles with $D=100-200$ $\mu \mathrm{m}$ (Leinert \& Grün 1990; Love \& Brownlee 1993). Also, equation (A15) with $D_{\text {typ }}=500 \mu \mathrm{m}$, $D_{c c}(D) / D=\left(10^{-4}\right)^{1 / 3}$, and $q=11 / 6$, predicts that the collisional lifetime of large bodies in the asteroid belt should be:

$$
t_{\text {coll }} \approx 10^{9} \sqrt{D}
$$

where $t_{\text {coll }}$ is given in years, and $D$ in $\mathrm{km}$. Since the solar system is $\sim 4.5 \times 10^{9}$ years old, this implies that asteroids larger than $\sim 20 \mathrm{~km}$ should be primordial asteroids; this is in agreement with more accurate models of the observed size distribution of these asteroids (Durda et al. 1998).

\footnotetext{
${ }^{3}$ If the particles had circular orbits, equation (28) means a spherical shell of width $d r$, the volume of which is $\propto r^{2} d r$, would contain a number of particles that is $\propto r d r$ (see e.g., Gorkavyi et al. 1997).
} 


\subsection{Division of a Disk into Particle Categories}

Disk particles of different sizes can be categorized according to the dominant physical processes affecting their evolution. Particles in the different categories have different lives; i.e., the way they are created, their dynamical evolution, and the way they are eventually destroyed, are all different. Each of a disk's categories has a different spatial distribution; which of these categories dominates the disk's observable structure depends on the relative contribution of each to the disk's cross-sectional area (see $\$ 2.3)$.

\subsubsection{Category Definitions}

A disk's largest particles, those with $\beta<0.1$, have orbital elements that are initially the same as, or at least very similar to, those of their parents. Of these large particles, only those with $\beta<\beta_{p r}$ suffer no significant P-R drag evolution to their orbits in their lifetime. These truly large particles continue on the same orbits as those of their ancestors until they collide with a particle large enough to cause a catastrophic collision; the resulting collisional fragments populate the collisional cascade. The spatial distribution of these "large" particles in a disk is its base distribution. The spatial distributions of a disk's smaller particles can only be understood in terms of how they differ from the disk's base distribution.

Disk particles with $\beta_{p r}<\beta<0.1$, spiral in from their parent's orbits due to P-R drag, so that they are closer to the star than their parents by the time of their demise (which could be caused

either by collisions or by evaporation close to the star); the spatial distribution of these "P-R drag affected" particles in a disk differs from the disk's base distribution in that it extends closer in to the star. The orbits of particles with $0.1<\beta<0.5$ also undergo significant P-R drag evolution before their demise, but their original orbits are already different from those of their parents; the spatial distribution of these " $\beta$ critical" particles in a disk extends both further out, and further in, from the disk's base distribution. Particles with $\beta>0.5$ leave their parents on hyperbolic orbits, and so are quickly lost from the system; the spatial distribution of these " $\beta$ meteoroids" in a disk extends further out, but not further in, from the disk's base distribution.

Thus, a disk comprises four particle categories, each of which has a different spatial distribution, although all are inextricably linked to that of the large particles through the collisional cascade. For disks that have $\beta_{p r}>0.5$, however, i.e., those with

$$
\tau_{e f f}(r)>10^{-4} \sqrt{\left(M_{\star} / M_{\odot}\right)\left(a_{\oplus} / r\right)},
$$

there is no significant P-R drag evolution of any of its constituent particles. Such disks comprise just three categories, since the P-R drag affected category is empty. 


\subsubsection{Category Cross-Sectional Area}

The size distributions of a disk's large particles, and its P-R drag affected particles, were discussed in $\S \S 3.1 .2$ and 3.1.3. These discussions imply that the cross-sectional area of a disk in which there is a population of P-R drag affected particles (see eq. [31]) is dominated by particles with $D \approx D_{p r}$ (eq. [27). As a first-cut approximation, the size distribution of a disk in which there are no P-R drag affected particles follows equation (15) from $D_{\max }$ down to $D_{\min }=D(\beta=0.5)$; however, since a particle's catastrophic collision rate is affected by the size distribution of particles smaller than itself (Durda \& Dermott 1997; eq. [A4]), this distribution cannot be expected to hold all the way down to $D(\beta=0.5)$. This means that the cross-sectional area of such a disk is concentrated in its smallest particles, and the contribution of $\beta$ critical particles to the disk's total cross-sectional area is given by

$$
d \sigma / \sigma_{\text {tot }}=\left[D^{5-3 q}\right]_{D(\beta=0.1)}^{D(\beta=0.5)} /\left[D^{5-3 q}\right]_{D_{\max }(\beta=0.5)}^{(\beta=}
$$

e.g., if $q=11 / 6$, and $\beta \propto 1 / D$, this means that half of the disk's cross-sectional area comes from its $\beta$ critical particles.

Since $\beta$ meteoroids have hyperbolic orbits, they are expected to contribute little to a disk's cross-sectional area unless they are produced at a high enough rate to replenish their rapid loss from the system. This could be the case if the disk was very dense, since material would pass quickly through the cascade; such a disk would undergo considerable mass loss. An estimate of how dense the disk would have to be for this to be the case depends on the assumptions made about the physics of collisions between small particles. For heuristic purposes, it is assumed here that the total cross-sectional area of $\beta$ meteoroids created by the collisional break-up of a parent body is comparable to that of the parent itself; this is probably an underestimate if the collision is destructive, but an overestimate if the collision is erosive. If this were the case, then the disk's $\beta$ meteoroids would dominate a disk's cross-sectional area only if their lifetime, which is of the order of the orbital period of their parents, is longer than the lifetime of these parents, which can be approximated by equation (16), i.e., only if

$$
\tau_{\text {eff }}(r)>0.1
$$

In conclusion, from a theoretical stand-point, there are few solid assumptions that can be made about a disk's size distribution, other than that the denser a disk is, the smaller the diameter of particles that its cross-sectional area is concentrated in.

\subsection{The Perturbed Dynamic Disk}

In addition to the physical processes described in $\delta 3.1$, the particles of the dynamic disk described in this section are affected by a number of perturbing processes; these produce subtle, 
but perhaps observable, changes in a disk's structure. The dominant perturbing processes in the zodiacal cloud are the secular and resonant gravitational perturbations of the planets. Secular perturbations are discussed in $\S$. Resonant perturbations give rise to planetary resonant rings: a planet's resonant ring is an asymmetric circumstellar ring of material that co-orbits with the planet as a result of the resonant trapping of particles into the planet's exterior mean motion resonances (not to be confused with circumplanetary dust rings). There is both observational and theoretical evidence for the existence of the Earth's resonant ring (Dermott et al. 1994; Reach et al. 1995); many of the observed Kuiper belt objects are trapped in 2:3 resonance with Neptune (Jewitt 1999), thus forming Neptune's resonant ring (Malhotra 1995).

Other possible perturbing processes include: stellar wind forces, that, at least for dust in the solar system, effectively increase the value of $\beta$ for P-R drag (e.g., Leinert \& Grün 1990); Lorentz forces acting on charged particles, that, while negligible for particles in the inner solar system, are increasingly important for particles at distances further from the Sun (e.g., Kimura \& Mann 1998); interactions with dust from the interstellar medium (e.g., Artymowicz \& Clampin 1997); the sublimation of icy dust grains, which is one of the mechanisms that has been suggested as the cause of the inner hole in the HR 4796 disk (Jura et al. 1998); and the self gravity of a massive disk, which could have played an important role in determining the evolution of the primordial Kuiper belt (Ward \& Hahn 1998).

\section{Structure of a Secularly Perturbed Disk}

The orbit of a particle in a circumstellar disk that is in a system in which there are one or more massive perturbers is inevitably affected by the gravitational perturbations of these bodies. The consequent evolution of the particle's orbit can be used to obtain a quantitative understanding of the effect of these perturbations on the structure of the disk.

\subsection{Secular Perturbation Theory}

\subsubsection{Perturbation Equations}

The gravitational forces from a planetary system that act to perturb the orbit of a particle in the system can be decomposed into the sum of many terms that are described by the particle's disturbing function, $R$. The long-term average of these forces are the system's secular perturbations, and the terms of the disturbing function that contribute to these secular perturbations, $R_{s e c}$, can be identified as those that do not depend on the mean longitudes of either the planets or the particle (the other forces having periodic variations).

Consider a particle that is orbiting a star of mass $M_{\star}$, that also has $N_{p l}$ massive, perturbing, bodies orbiting it. This particle has a radiation pressure force acting on it represented by $\beta$, 
and its orbit is described by the elements $a, e, I, \Omega$ and $\tilde{\omega}$. To second order in eccentricities and inclinations, the secular terms in the particle's disturbing function are given by (Brouwer \& Clemence 1961; Dermott et al. 1985; Dermott \& Nicholson 1986; Murray \& Dermott 1999):

$$
R_{s e c}=n a^{2}\left[\frac{1}{2} A\left(e^{2}-I^{2}\right)+\sum_{j=1}^{N_{p l}}\left[A_{j} e e_{j} \cos \left(\tilde{\omega}-\tilde{\omega}_{j}\right)+B_{j} I I_{j} \cos \left(\Omega-\Omega_{j}\right)\right]\right],
$$

where $n=\left(2 \pi / t_{\text {year }}\right) \sqrt{\left(M_{\star} / M_{\odot}\right)(1-\beta)\left(a_{\oplus} / a\right)^{3}}$ is the mean motion of the particle in $\mathrm{rad} / \mathrm{s}$, $t_{\text {year }}=2 \pi / \sqrt{G M_{\odot} / a_{\oplus}^{3}}=3.156 \times 10^{7} \mathrm{~s}$ is one year measured in seconds, and

$$
\begin{aligned}
A & =+\frac{n}{4(1-\beta)} \sum_{j=1}^{N_{p l}}\left(\frac{M_{j}}{M_{\star}}\right) \alpha_{j} \bar{\alpha}_{j} b_{3 / 2}^{1}\left(\alpha_{j}\right), \\
A_{j} & =-\frac{n}{4(1-\beta)}\left(\frac{M_{j}}{M_{\star}}\right) \alpha_{j} \bar{\alpha}_{j} b_{3 / 2}^{2}\left(\alpha_{j}\right), \\
B_{j} & =+\frac{n}{4(1-\beta)}\left(\frac{M_{j}}{M_{\star}}\right) \alpha_{j} \bar{\alpha}_{j} b_{3 / 2}^{1}\left(\alpha_{j}\right),
\end{aligned}
$$

where $\alpha_{j}=a_{j} / a$ and $\bar{\alpha}_{j}=1$ for $a_{j}<a$, and $\alpha_{j}=\bar{\alpha}_{j}=a / a_{j}$ for $a_{j}>a$, and $b_{3 / 2}^{s}\left(\alpha_{j}\right)=(\pi)^{-1} \int_{0}^{2 \pi}\left(1-2 \alpha_{j} \cos \psi+\alpha_{j}^{2}\right)^{-3 / 2} \cos s \psi d \psi$ are the Laplace coefficients $(s=1,2) . A, A_{j}$ and $B_{j}$ are in units of $\mathrm{rad} / \mathrm{s}$, and $R_{\text {sec }}$ is in units of $\mathrm{m}^{2} / \mathrm{s}^{2}$.

The effect of these perturbations on the orbital elements of the particle can be found using Lagrange's planetary equations (Brouwer \& Clemence 1961; Murray \& Dermott 1999). The semimajor axis of the particle remains constant, $\dot{a}_{s e c}=0$, while the variations of its eccentricity and inclination are best described when coupled with the variations of its longitude of pericenter and ascending node using the variables defined by its complex eccentricity, $z$, and complex inclination, $y$ :

$$
\begin{aligned}
& z=e * \exp i \tilde{\omega} \\
& y=I * \exp i \Omega
\end{aligned}
$$

where $i^{2}=-1$. Using these variables Lagrange's planetary equations give the orbital element variations due to secular perturbations as:

$$
\begin{aligned}
& \dot{z}_{s e c}=+i A z+i \sum_{j=1}^{N_{p l}} A_{j} z_{j}, \\
& \dot{y}_{s e c}=-i A y+i \sum_{j=1}^{N_{p l}} B_{j} y_{j},
\end{aligned}
$$

where $z_{j}$ and $y_{j}$ are the complex eccentricities and inclinations of the perturbers, which have a slow temporal variation due to the secular perturbations of the perturbers on each other Brouwer 
\& Clemence 1961; Murray \& Dermott 1999):

$$
\begin{aligned}
& z_{j}(t)=\sum_{k=1}^{N_{p l}} e_{j k} * \exp i\left(g_{k} t+\beta_{k}\right), \\
& y_{j}(t)=\sum_{k=1}^{N_{p l}} I_{j k} * \exp i\left(f_{k} t+\gamma_{k}\right),
\end{aligned}
$$

where $g_{k}$ and $f_{k}$ are the eigenfrequencies of the perturber system, the coefficients $e_{j k}$ and $I_{j k}$ are the corresponding eigenvectors, and $\beta_{k}$ and $\gamma_{k}$ are constants found from the initial conditions of the perturber system.

\subsubsection{Solution to Perturbation Equations}

Ignoring the evolution of a particle's orbital elements due to P-R drag, equations (37a) and (37b) can be solved to give the secular evolution of the particle's instantaneous complex eccentricity and inclination (a.k.a. the particle's osculating elements). This secular evolution is decomposed into two distinct time-varying elements — the "forced", subscript $f$, and "proper", subscript $p$, elements — that are added vectorially in the complex planes (see e.g., Fig. 2a):

$$
\begin{aligned}
& z(t)=z_{f}(t)+z_{p}(t)=\sum_{k=1}^{N_{p l}}\left[\frac{\sum_{j=1}^{N_{p l}} A_{j} e_{j k}}{g_{k}-A}\right] * \exp i\left(g_{k} t+\beta_{k}\right)+e_{p} * \exp i\left(+A t+\beta_{0}\right), \\
& y(t)=y_{f}(t)+y_{p}(t)=\sum_{k=1}^{N_{p l}}\left[\frac{\sum_{j=1}^{N_{p l}} B_{j} I_{j k}}{f_{k}+A}\right] * \exp i\left(f_{k} t+\gamma_{k}\right)+I_{p} * \exp i\left(-A t+\gamma_{0}\right),
\end{aligned}
$$

where $e_{p}, \beta_{0}$, and $I_{p}, \gamma_{0}$ are determined by the particle's initial conditions.

These equations have simple physical and geometrical interpretations. A particle's forced elements, $z_{f}$ and $y_{f}$, depend only on the orbits of the perturbers in the system (that have a slow secular evolution, eqs. [38a] and 38b]), as well as on the particle's semimajor axis (which has no secular evolution). Thus, at a time $t_{0}$, a particle that is on an orbit with a semimajor axis $a$, has forced elements imposed on its orbit by the perturbers in the system that are defined by $z_{f}\left(a, t_{0}\right)$ and $y_{f}\left(a, t_{0}\right)$. The contribution of the particle's proper elements to its osculating elements, $z\left(t_{0}\right)$ and $y\left(t_{0}\right)$, is then given by: $z_{p}\left(t_{0}\right)=z\left(t_{0}\right)-z_{f}\left(a, t_{0}\right)$ and $y_{p}\left(t_{0}\right)=y\left(t_{0}\right)-y_{f}\left(a, t_{0}\right)$; thus defining the particle's proper eccentricity, $e_{p}$, and proper inclination, $I_{p}$, which are its fundamental orbital elements (i.e., those that the particle would have if there were no perturbers in the system), as well as the orientation parameters $\beta_{0}$ and $\gamma_{0}$. Since both the forced elements, and the osculating elements, of collisional fragments are the same as those of their parent (apart from fragments with $\beta>0.1$ ), particles from the same family have the same proper elements, $e_{p}$ and $I_{p}$.

The evolution of a particle's proper elements is straight-forward - they precess around circles of fixed radius, $e_{p}$ and $I_{p}$, at a constant rate, $A$, counterclockwise for $z_{p}$, clockwise for $y_{p}$. The 
secular precession timescale depends only on the semimajor axis of the particle's orbit:

$$
t_{\text {sec }}=2 \pi / A t_{\text {year }},
$$

where $t_{s e c}$ is given in years, and $A$ is given in equation (35a); secular perturbations produce long period variations in a particle's orbital elements (e.g., $t_{\text {sec }}=O(0.1 \mathrm{Myrs})$ in the asteroid belt). The centers of the circles that the proper elements precess around are the forced elements (see e.g., Fig. 2a). Actually the forced elements vary on timescales that are comparable to the precession timescale (eq. 40]); thus, it might appear ambitious to talk of the precession of a particle's osculating elements around circles when its real evolutionary track in the complex eccentricity and complex inclination planes may not be circular at all. The reason it is presented as such is that at any given time, all of the particles at the same semimajor axis precess (at the same rate) around the same forced elements on circles of different radii, and this has consequences for the global distribution of orbital elements (see \$4.2.1).

There are two things that are worth mentioning now about a particle's forced elements. If there is just one perturber in the system, $N_{p l}=1$, its complex eccentricity and complex inclination do not undergo any secular evolution, and the forced elements imposed on a particle in the system are not only constant in time, but also independent of the mass of the perturber:

$$
\begin{aligned}
z_{f} & =\left[b_{3 / 2}^{2}\left(\alpha_{j}\right) / b_{3 / 2}^{1}\left(\alpha_{j}\right)\right] e_{j} * \exp i \tilde{\omega}_{j}, \\
y_{f} & =I_{j} * \exp i \Omega_{j} .
\end{aligned}
$$

This implies that a body of low mass, such as an asteroid, has as much impact on a particle's orbit as a body of high mass, such as a Jupiter mass planet. The perturbations from a smaller perturber, however, produce longer secular precession timescales (eq. [40]):

$$
t_{s e c}=4\left[\alpha_{j} \bar{\alpha}_{j} b_{3 / 2}^{1}\left(\alpha_{j}\right)\left(a_{\oplus} / a\right)^{3 / 2}\left(M_{j} / M_{\star}\right) \sqrt{M_{\star} / M_{\odot}}\right]^{-1} ;
$$

they would also be similar in magnitude to those of the disk's self-gravity, which in that case could no longer be ignored. If there is more than one perturber in the system, $N_{p l}>1$, then particles on orbits for which their precession rate equals one of the system's eigenfrequencies $\left(A=g_{k}\right.$, or $-A=f_{k}$ ) have infinite forced elements imposed on their orbits, and so are quickly ejected from such a "secular resonance" region.

The solution given by equations (39a) and (39b) accounts for the fact that small particles see a less massive star due to the action of radiation pressure, but not for the P-R drag evolution of their orbits; the solution for these particles is discussed in Appendix B. Also, the perturbation theory of $\$ 4.1 .1$ is only valid for particles with small eccentricities; i.e., it is not valid for the evolution of a disk's $\beta$ critical particles, or its $\beta$ meteoroids. However, if the evolution of a disk's $\beta$ critical particles is affected by secular perturbations (i.e., if their lifetime is longer than the secular timescale), then it is probably also affected by P-R drag (i.e., their lifetime is probably also longer than the P-R drag timescale), in which case the disk's $\beta$ critical particles do not contribute much to its observable structure ( $\$ \$ 3.1 .3$ and 3.2 .2 ). There is no secular evolution to the orbits of $\beta$ meteoroids because of their short lifetimes. 


\subsection{Offset and Warp}

The effect of secular perturbations on the structure of a disk can be understood by considering the effect of the secular evolution of the constituent particles' orbits on the distribution of their

orbital elements. The perturbation equations of $\$ 4.1 .1$ show that secular pertubations affect only the distribution of disk particles' complex eccentricities, $n(z)$, and complex inclinations, $n(y)$, while having no effect on their size distribution (and hence the division of the disk into its particle categories), or on their semimajor axis distribution (and hence the disk's large-scale radial distribution).

\subsubsection{Offset and Plane of Symmetry of Family Material}

Consider the family of collisional fragments originating from a primordial body, the orbital elements of which were described by $a, e_{p}$, and $I_{p}$. Here we consider only fragments that are unaffected by P-R drag (those that are affected by P-R drag are discussed in Appendix B).

Large $(\beta<0.1)$ Fragments The orbital elements of the largest fragments, those with $\beta<0.1$, created in the break-up of the primordial body are initially very close to those of the primordial body; they do not have identical orbits due to the velocity dispersion imparted to the fragments in the collision. The forced elements imposed on the orbits of all of these collisional fragments are the same as those imposed on the primordial body. The secular evolution of their osculating complex eccentricities (eq. [39a]) and complex inclinations (eq. [39b]), is to precess about the forced elements (which are also varying with time), but at slightly different rates (due to their slightly different semimajor axes). A similar argument applies for all particles created by the collisional break-up of these fragments. Thus, after a few precession timescales, the complex eccentricities and complex inclinations of the collisional fragments of this family lie evenly distributed around circles that are centered on $z_{f}(a, t)$ and $y_{f}(a, t)$, and that have radii of $e_{p}$ and $I_{p}$ (e.g., their complex eccentricities lie on the circle shown in Fig. 2a), while their semimajor axes are all still close to $a$. This is seen to be the case in the asteroid belt: there are families of asteroids that have similar $a, e_{p}$, and $I_{p}$, that are the collisional fragments resulting from the break-up of a much larger asteroid (Hirayama 1918).

The distribution of the complex eccentricities, $n(z)$, of these particles, has a distribution of pericenters that is biased towards the orientation in the disk that is defined by $\tilde{\omega}_{f}$. The consequence of this biased orbital element distribution on the spatial distribution of this family material is best described with the help of Fig. 2 $\mathrm{b}$. This shows a face-on view (i.e., perpendicular to the plane of symmetry), of the family material in orbit around a star $S$. The resulting disk is made up of particles on orbits that have the same $a, e_{f}, \tilde{\omega}_{f}$, and $e_{p}$, but random $\tilde{\omega}_{p}$. The contribution of each particle to the spatial distribution of material in the disk can be described by the elliptical ring of material coincident with the particle's orbit (see $\$ 3.1 .1$ ). In Fig. $2 \mathrm{~b}$, these 
elliptical rings have been represented by uniform circles of radius $a$, with centers that are offset by ae in a direction opposite to the pericenter direction, $\tilde{\omega}$ (this is a valid approximation to first order in the particles' eccentricities); a heavy line is used to highlight the orbital ring with a pericenter located at $P$, and a displaced circle center located at $D$, where $D P=a$. The vector $S D$ can be decomposed into its forced and proper components; this is shown by the triangle $S C D$, where $S D=a e, S C=a e_{f}$, and $C D=a e_{p}$ (there is a similar triangle in Fig. 2a). Given that the distribution of $\tilde{\omega}_{p}$ is random, it follows that the distribution of the rings' centers, $D$, for the family disk are distributed on a circle of radius $a e_{p}$ and center $C$. Thus, the family forms a uniform torus of inner radius $a\left(1-e_{p}\right)$ and outer radius $a\left(1+e_{p}\right)$ centered on a point $C$ displaced from the star $S$ by a distance $a e_{f}$ in a direction away from the forced pericenter, $\tilde{\omega}_{f}$ (Dermott et al. 1985; Dermott et al. 1998).

The distribution of the complex inclinations, $n(y)$, of these particles, is also the distribution of their orbital planes. Changing the reference plane relative to which the particles' orbital inclinations are defined to that described by $y_{f}$, shows that the secular complex inclination distribution of this family material leads to a disk that is symmetrical about the $y_{f}$ plane; the flaring of this disk is described by $I_{p}$.

Small $(\beta>0.1)$ Fragments Since the small particles in this family originate from the larger particles, the orbital elements of the parents of the small particles have the same $a, e_{p}$ and $I_{p}$ (they also have the same $z_{f}$ and $y_{f}$ ), but random $\tilde{\omega}_{p}$ and $\Omega_{p}$. Consider the $\beta$ critical particles that are produced at the same time from the population of family particles that have the same $\tilde{\omega}_{p}$ and $\Omega_{p}$ at that time; this parent population is spread out along the orbit defined by the elements $a$,

$e$, and $\tilde{\omega}$, which could be one of the rings shown in Fig. $2 \mathrm{~b}$. The average pericenter orientation of these $\beta$ critical particles, $\left\langle\tilde{\omega}^{\prime}\right\rangle$, is the same as that of the orbit of the larger particles, $\tilde{\omega}$ (obvious because of the symmetry of eq. [21] with respect to $f$ ); their pericenter locations are also the same (eq. [22]). Thus, the ring shown in Fig. 2b defines the inner edge of the disk of $\beta$ critical particles created in the break-up of large particles on this ring; i.e., their disk is offset by an amount ae in the $\tilde{\omega}$ direction. Consequently, the inner edge of the disk of $\beta$ critical particles created in the break-up of all large particles in this family is offset by an amount $a e_{f}$ in the $\tilde{\omega}_{f}$ direction. This disk has the same plane of symmetry as the families' large particles, since all particle categories from the same family have the same distribution of orbital planes, $n(y)$. Similar arguments apply for the families' $\beta$ meteoroids.

\subsubsection{Offset and Warp of Whole Disk}

The disks of material from all of the families that have the same semimajor axis, or equivalently that are at the same distance from the star, have the same offset inner edge, and the same plane of symmetry. This is because their large particles have the same forced elements imposed on their orbits. The complex eccentricities and complex inclinations of the large particles 
of all of these families lie evenly distributed around circles with the same centers, $z_{f}$ and $y_{f}$, but with a distribution of radii, $n\left(e_{p}\right)$ and $n\left(I_{p}\right)$, that are the distributions of the proper elements of these families (defining the width and flaring of the torus consisting of these families' material). The whole disk is made up of families with a range of semimajor axes; the families that are at different semimajor axes can have different forced elements imposed on their orbits, and in view of the proper element distributions in the asteroid belt and in the Kuiper belt, they can also have different distributions of proper elements.

If the forced eccentricity imposed on the disk is non-zero, which it is if there is at least one perturber in the system that is on a non-circular orbit (eqs. [39a] and 419]), then the disk's center of symmetry is offset from the star. If the forced inclination imposed on the disk is different for families at different semimajor axes, which it is if there are two or more perturbers in the system that are moving on orbits that are not co-planar (eqs. [39b] and 41b]), then the disk's plane of symmetry varies with distance from the star; i.e., the disk is warped.

\subsubsection{Physical Understanding of Offset and Warp}

There is also a physical explanation for the secular perturbation asymmetries. The secular perturbations of a massive body are equivalent to the gravitational perturbations of the elliptical ring that contains the mass of the perturber spread out along its orbit, the line density of which varies inversely with the speed of the perturber in its orbit (Gauss' averaging method, Brouwer \& Clemence 1961; Murray \& Dermott 1999). The ring's elliptical shape, as well as its higher line density at the perturber's apocenter, mean that the center of mass of the "star-ring" system is shifted from the star towards the perturber's apocenter. The focus of the orbits of particles in such a system is offset from the star; i.e., the center of symmetry of a disk in this system is offset from the star. The gravitational perturbations of the ring also point to the plane coincident with the perturber's orbital plane. In systems with two or more perturbers, the system's plane of symmetry (that in which the perturbing forces out of this plane cancel) varies with distance from the star; i.e., a disk in such a system is warped.

\subsection{Observational Evidence of Offset and Warp in the Zodiacal Cloud}

Mid-IR geocentric satellite observations (such as the IRAS, COBE, and ISO observations) are dominated by the thermal emission of the zodiacal cloud's P-R drag affected particles in all directions except that of the galactic plane (Leinert \& Grün 1990). Such observations contain detailed information about the spatial structure of the zodiacal cloud, especially since their observing geometry changes throughout the year as the Earth moves around its orbit. Since there are 9 massive perturbers in the solar system, the resulting secular perturbation asymmetries should be apparent in the IRAS, COBE, and ISO data-sets (see Appendix B for a discussion of 
why the distribution of a disk's P-R drag affected particles should also contain the signatures of the system's secular perturbations).

Fig. 3a shows COBE observations of the sum of the brightnesses in the $25 \mu \mathrm{m}$ waveband at the north and south ecliptic poles, $(N+S) / 2$ (Dermott et al. 1999), where there is no contamination from the galactic plane. If the zodiacal cloud was rotationally symmetric with the Sun at the center, then the cross-sectional area density of particles in the near Earth region would vary according to $\sigma(r, \theta, \phi) \propto r^{-\nu} f(\phi)$, where $\nu$ is a constant. Because the Earth's orbit is eccentric, geocentric observations sample the zodiacal cloud at different radial distances from the Sun. Thus, the minimum of the $(N+S) / 2$ observation is expected to occur either at the Earth's aphelion, $\lambda_{\oplus}=282.9^{\circ}$, or perihelion, $\lambda_{\oplus}=102.9^{\circ}$, depending on whether $\nu>1$ or $\nu<1$, which is determined by the collisional evolution of particles in the near-Earth region (e.g., Leinert \& Grün 1990 discuss the observational evidence and conclude that $\nu \approx 1.3$ as found by the Helios zodiacal light experiment). However, the minimum in the $25 \mu \mathrm{m}$ waveband observations occurs at $\lambda_{\oplus}=224^{\circ}$, and a similar result is found in the $12 \mu \mathrm{m}$ waveband. This is expected only if the Sun is not at the center of symmetry of the zodiacal cloud. Parametric models of the zodiacal cloud have also shown the need for an offset to explain the observations (e.g., Kelsall et al. 1998).

Fig. $3 \mathrm{~b}$ shows the variation of the brightnesses of the ecliptic poles with ecliptic longitude of the Earth (Dermott et al. 1999). The north and south polar brightnesses are equal when the Earth is at either the ascending or descending node of the local (at $1 \mathrm{AU}$ ) plane of symmetry of the cloud, giving an ascending node of $\Omega_{a s c}=70.7 \pm 0.4^{\circ}$. However, COBE observations of the latitudes of the peak brightnesses of the zodiacal cloud measured in the directions leading and trailing the Earth's orbital motion give $\Omega_{a s c}=58.4^{\circ}$ (Dermott et al. 1996). Since such observations sample the cloud external to $1 \mathrm{AU}$, this implies that the plane of symmetry of the zodiacal cloud varies with heliocentric distance, i.e., that the zodiacal cloud is warped.

To observe the zodiacal cloud's offset and warp asymmetries, an observer outside the solar system, would, at the very least, need an observational resolution greater than the magnitude of these asymmetries; e.g., to observe the offset asymmetry, the observer needs a resolution of $>\left(a e_{f} / a_{\oplus}\right) / R_{\odot}$ arcseconds, where the distance from the observer to the Sun, $R_{\odot}$, is measured in pc. An offset would be more observable in a disk such as HR 4796 due to its central cavity, since this causes a brightness asymmetry in the emission from the inner edge of the disk.

\section{The Secularly Perturbed HR 4796 Disk Model}

This section describes our model of the HR 4796 disk that accounts for the brightness distribution seen in the IHW18 waveband observation of T99 (Fig. Ba). There are three components of the observation that had to be included in the model (\$2): the disk's structure, $\sigma(r, \theta, \phi)$, defined in $\S 2.2$; the combination of the optical properties and the size distribution of the disk's particles given by $P(\lambda, r)$, defined in $\S 2.3$; and the disk's orientation. While the modeling 
techniques used here are new to the study of circumstellar disks, they have already been widely used to study the observed structure of the zodiacal cloud (see, e.g., Dermott et al. 1994; Grogan et al. 1997), and so can be used with a certain degree of confidence.

\subsection{Model of Offset Disk Structure, $\sigma(r, \theta, \phi)$}

The disk's structure was modeled as that of a secularly perturbed dynamic disk. A combination of the theory of $\S \S 3$ and 4 , and inferences from the observation (Fig. 8a), was used to parameterize the distribution of the orbital elements of the disk's large particles ( $\$[$ 5.1.1). This was used to create parameterized models of the spatial distribution of these large particles (\$5.1.2), that could then be compared with the observed spatial distribution. Since the observed spatial distribution is that of the "emitting" particles, the underlying assumption is that these emitting particles either are the disk's large particles, or that their spatial distribution is the same as that of the disk's large particles (whether they are large or not). The implications of this assumption are discussed in the interpretation of the model (s 6 ).

\subsubsection{Distribution of Orbital Elements, $\sigma(a, e, I, \Omega, \tilde{\omega})$}

The quintessentially secular part of the distribution of the orbital elements of the large particles in a secularly perturbed disk is the distributions of their complex eccentricities, $n(z)$, and complex inclinations, $n(y)$. For a particle with an orbit of a given semimajor axis, $a$, its complex eccentricity, $z$, and complex inclination, $y$, are the addition of forced elements, $z_{f}(a)$ and $y_{f}(a)$, to proper elements that have $\tilde{\omega}_{p}$ and $\Omega_{p}$ chosen at random, while $e_{p}$ and $I_{p}$ are chosen from the distributions $n\left(e_{p}\right)$ and $n\left(I_{p}\right)$.

Since there is insufficient information available to determine the variation of the forced and proper elements with semimajor axis in this disk, they were assumed to be constant across the disk. The forced elements were left as model variables: the forced eccentricity, $e_{f}$, defines the magnitude of the offset asymmetry in the disk model; the forced pericenter orientation, $\tilde{\omega}_{f}$, defines the orientation of this asymmetry; and the forced inclination, $y_{f}$, defines the plane of symmetry of the disk model. When creating a disk model, both $\tilde{\omega}_{f}$ and $y_{f}$ were set to zero; these were incorporated later into the description of the disk's orientation to our line of sight (see $\$ 5.3)$. The distributions of the proper eccentricities, $n\left(e_{p}\right)$, and proper inclinations, $n\left(I_{p}\right)$, of particles in the disk model were taken to be like those of the main-belt asteroids with absolute magnitudes $H<11$ (Bowell 1996). These large asteroids constitute a bias-free set (Bowell 1996) and have mean proper eccentricities and proper inclinations of $\left\langle e_{p}\right\rangle=0.130$ and $\left\langle I_{p}\right\rangle=10.2^{\circ}$. Not enough Kuiper belt objects have been discovered yet to infer a bias-free distribution for their orbital elements.

The distribution of the semimajor axes, $n(a)$, of particles in the disk defines its radial distribution. There is no way of guessing this distribution from theoretical considerations, since it 
depends on the outcome of the system's planetary formation process, which varies from system to system (compare the distribution of the solar system's planets, and its disk material, with those found in exosolar systems, e.g., Backman \& Paresce 1993; Marcy \& Butler 1998). Thus, it had to be deduced purely observationally. Fig. 8a shows that the disk has an inner edge, inside of which there is a negligible amount of dust; this was modeled as a sharp cut-off in the distribution of semimajor axes at $a_{\text {min }}$, a model variable. The observation also shows that the disk has an outer edge at $\sim 130 \mathrm{AU}$; this was modeled as a sharp cut-off in the distribution of semimajor axes at $a_{\max }=130 \mathrm{AU}$ (this is a non-critical parameter, since particles near the outer edge of the disk contribute little to the observation, see $\$ 5.2 .3$ ). The distribution between $a_{\min }$ and $130 \mathrm{AU}$ was taken as $n(a) \propto a^{\gamma}$, where $n(a) d a$ is the number of particles on orbits with semimajor axes in the range $a \pm d a / 2$, and $\gamma$ is a model variable. To get an idea of the radial distribution resulting from this semimajor axis distribution, consider that if the particles had zero eccentricity, this distribution would result in a volume density (number of particles per unit volume) distribution that is $\propto r^{\gamma-2}$ (since the number of particles in a spherical shell of width $d r$, the volume of which is $\propto r^{2} d r$, would contain a number of particles that is $\left.\propto r^{\gamma} d r\right)$.

\subsubsection{Conversion to Spatial Distribution, $\sigma(r, \theta, \phi)$}

Disk models were created from the orbital element distribution of $\$ 5.1 .1$ using the "SIMUL" program; SIMUL was developed by the solar system dynamics group at the University of Florida (Dermott et al. 1992). A disk model is a large three-dimensional array, $\sigma(r, \theta, \phi)$, that describes the spatial distribution of the cross-sectional area of material in the disk model per unit volume binned in: $r$, the radial distance from the star; $\theta$, the longitude relative to an arbitrary direction (set here as the forced pericenter direction, $\tilde{\omega}_{f}$ ); and $\phi$, the latitude relative to an arbitrary plane (set here as the forced inclination, $y_{f}$, or symmetry, plane). SIMUL creates a disk model by taking the total cross-sectional area of material in the disk (specified by the model variable $\sigma_{\text {tot }}$ ), and dividing it equally among a large number of orbits ( 5 million in this case), the elements of each of which are chosen randomly from the specified distribution ( $\$$.1.1). The disk model is populated by considering the contribution of each orbit to the cross-sectional area density in each of the cells it crosses.

The spatial distribution of material in one of our models of the HR 4796 disk can be described by the three variables $a_{\text {min }}, \gamma$, and $e_{f} ; \sigma_{t o t}$ simply scales the amount of material in the model, and $\tilde{\omega}_{f}$ and $y_{f}$ describe the orientation of the disk to our line of sight. Fig. 4 is a plot of the surface density of material in a disk model with $a_{\text {min }}=62 \mathrm{AU}, e_{f}=0.02$, and $\gamma=-2$ (this is our final model of $\$ 5.4$. This illustrates how the specified distribution of orbital elements affects the spatial distribution of material in the disk model: the sharp cut-off in semimajor axes at $a_{\text {min }}$ determines the radial location of the inner hole, which has a sloping cut-off in $r$ due to the particles' eccentricities; as predicted in $\$ 4.2 .1$, particles at the inner edge of the disk in the forced pericenter direction are closer to the star than those in the forced apocenter direction by 
$\sim 2 a_{\min } e_{f}$; the distribution of semimajor axes has produced a surface density distribution that is $\propto r^{\gamma-1}$, but only exterior to $70 \mathrm{AU}$.

\section{2. $\quad$ Model of $P(\lambda, r)$}

\subsubsection{Optical Properties of Disk Particles}

The optical properties of the disk particles were found assuming the particles to be made of astronomical silicate (Draine \& Lee 1984; Laor \& Draine 1993), a common component of interplanetary dust found in both the zodiacal cloud (Leinert \& Grün 1990) and exosolar systems (e.g., Telesco \& Knacke 1991; Fajardo-Acosta, Telesco, \& Knacke 1993; Sitko et al. 1999); such an assumption can be tested at a later date using spectroscopy to look for silicate features in the HR 4796 disk emission. Furthermore, the particles were assumed to be solid, spherical, and have a density of $\rho=2500 \mathrm{~kg} / \mathrm{m}^{3}$. Their optical properties were calculated using Mie theory, assuming that HR 4796A has a luminosity and temperature of $L_{\star}=21 L_{\odot}, T_{\star}=9500^{\circ} \mathrm{K}$ (Jura et al. 1998), and using for its spectrum, that of the A0V star Vega (Cohen 1999). In all calculations, the mass of HR 4796A was assumed to be $M_{\star}=2.5 M_{\odot}$ (Jayawardhana et al. 1998).

The properties of particles of different sizes, and at different distances from HR 4796A, are shown in Fig. 5. The temperatures of the particles are plotted in Figs. 5a and 5b. The form of Fig. Fa can be understood by consideration of equation (11), and the wavelengths at which the star and a particle, if it was a black body, emit most of their energy: $\lambda_{\star} \approx 2898 / T_{\star} \mu \mathrm{m}$, and $\lambda_{b b} \approx 2898 / T_{b b}=10 \sqrt{r / a_{\oplus}}\left(L_{\odot} / L_{\star}\right)^{0.25} \mu \mathrm{m}$. As a crude approximation, a particle with diameter $D$ has $Q_{a b s} \approx 1$ for $\lambda \ll \pi D$ and $Q_{a b s} \rightarrow 0$ for $\lambda \gg \pi D$. Thus, in terms of their thermal properties, disk particles can be divided into four categories: the largest particles, $D \gg \lambda_{b b} / \pi$, are efficient absorbers and emitters at all relevant wavelengths and so achieve nearly black body temperatures, $T_{b b}$; particles with $D \ll \lambda_{b b} / \pi$, are inefficient emitters at their black body temperature, and so need temperatures higher than $T_{b b}$ to re-radiate all of the incident energy; the smallest particles, $D \ll \lambda_{\star} / \pi$, are also inefficient absorbers at the stellar temperature, and so do not need as high temperatures as slightly larger particles to re-radiate the absorbed energy; and particles with $D \approx 20 \mu \mathrm{m}$ have temperatures below that of a black body - this is because these particles are super-efficient emitters at their black body temperatures (due to silicate resonances, $Q_{a b s}$ can go up as high as 2), and so need lower temperatures to re-radiate the incident energy. The form of Fig. 5b can be understood in the same way. The fall-off of a large (e.g., $D=1000 \mu \mathrm{m}$ ) particle's temperature with distance from HR 4796A is like that of a black body, i.e., $T \propto 1 / \sqrt{r}$, while the fall-off for smaller particles is not that steep because these particles emit less efficiently the further they are from the star (due to their lower temperatures, and consequently higher $\lambda_{b b}$ ); e.g., the fall-off for $D=2.5 \mu \mathrm{m}$ particles is $T \propto 1 / r^{0.34}$, which is close to the $1 / r^{1 / 3}$ fall-off expected for particles with an emission efficiency that decreases $\propto 1 / \lambda^{2}$ (e.g., Backman \& Paresce 1993).

More important observationally is the variation of the particles' $Q_{a b s}(\lambda, D) B_{\nu}[\lambda, T(D, r)]$, 
since this determines the contribution of a particle's thermal emission to the flux density received at the Earth (eqs. [3], [7] and [8]). This is plotted for $\lambda=18.2 \mu \mathrm{m}$ in Figs. 5c and 55d, and for $\lambda=10.8 \mu \mathrm{m}$ in Figs. Fe and Ff. The form of Figs. 5c and 5e can be explained in the same way that Fig. 5a was explained: all three figures have similar forms, which is to be expected since the particles' temperature also appears in $B_{\nu}$; Figs. 5c and 5e are, however, attenuated for $D \ll \lambda / \pi$, since these particles are inefficient emitters at that wavelength. The fall-off with distance of the different particles shown in Figs. 5d and $5 \mathrm{f}$ is due solely to their different temperature fall-offs (Fig. 5b); e.g., for $\lambda=18.2 \mu \mathrm{m}$, the approximate fall-off for $D=1000 \mu \mathrm{m}$ particles is $\propto 1 / r^{5.4}$, while that for $D=2.5 \mu \mathrm{m}$ particles is $\propto 1 / r^{2.6}$.

\subsubsection{Cross-sectional Area Distribution}

The definition of $P(\lambda, r)$ (eqs. [8] and [9]) shows that it is the convolution of $Q_{a b s} B_{\nu}$ (Fig. Fic $5 \mathrm{f})$ with the cross-sectional area distribution, $\bar{\sigma}(D, r)$. Since theoretical arguments cannot supply an accurate size distribution ( $\$ 3.2 .2)$, we use the assumption of equation (10), which is that $P(\lambda, r)$ is equal to the $Q_{a b s} B_{\nu}$ of particles in the disk with characteristic size, $D_{t y p}$. We further assume that this $D_{t y p}$ is constant across the disk in the IHW18 waveband; i.e., the brightness of a disk model observation in the IHW18 waveband is calculated using $P(\lambda, r)$ from the line on Fig. 5 c corresponding to $D_{t y p}$, where $D_{t y p}$ is a model variable. A qualitative understanding of $D_{t y p}$ comes from Fig. 5c: since $Q_{a b s} B_{\nu}$ is fairly flat for particles larger than $\sim 8 \mu \mathrm{m}$, an IHW18 waveband observation is dominated by those particles with the most cross-sectional area, unless there are a significant amount of particles smaller than $8 \mu \mathrm{m}$; Fig. 5ic also shows that the observation is unlikely to be dominated by particles smaller than $O(0.01 \mu \mathrm{m})$, unless their contribution to the disk's cross-sectional area is much higher than that of larger particles.

In addition to the IHW18 waveband, T99 observed HR 4796 in the $\mathrm{N}(\lambda=10.2 \mu \mathrm{m})$ waveband. The $\mathrm{N}$ band observations also show the double-lobed feature, but the inaccuracy of the subtraction of the image of HR 4796A from the observations means that they cannot be used to constrain the disk's structure. The $\mathrm{N}$ band observations can, however, be used to constrain the disk's brightness in this waveband; the brightness of a disk in different wavebands differs only in the factor $P(\lambda, r)$ (eq. [7]), and so the $\mathrm{N}$ band observation can be used to obtain information about the disk's size distribution. Since Figs. 5c and 5e have similar forms, the two observations should be dominated by the emission of similarly sized particles; i.e., they should have the same $D_{t y p}$, and show similar structures. The same characteristic particle size, $D_{t y p}$, was used to calculate the brightness of a disk model in both the IHW18 and N wavebands, using $P(\lambda, r)$ from the appropriate lines on the plots of Figs. 5 $\mathrm{d}$ and $5 \mathrm{f}$. There is just one $D_{t y p}$ that simultaneously matches the disk's observed brightnesses in both wavebands.

We made an initial estimate for $D_{t y p}$ based on the flux densities observed in the two wavebands: the flux densities of the disk in the IHW18 and N wavebands are 857 and 40 mJy, respectively (T99); i.e., the observed flux density ratio (N/IHW18) is $O(0.05)$. The expected flux 
density ratio of the two wavebands (the ratio of $P(\lambda, r)$ for different $D_{t y p}$ ) is plotted in Fig. 6a. Assuming the emission to arise mostly from particles near the inner edge of the disk, $r=60-80$ AU, Fig. 6a shows that the observed emission can be fairly well-constrained to come from particles with $D_{t y p}=2-3 \mu \mathrm{m}$.

\subsubsection{Pericenter Glow}

Fig. 7a shows a contour plot of an unsmoothed IHW18 waveband observation of the disk model of Fig. 4 viewed face-on (i.e., perpendicular to the disk's plane of symmetry, $y_{f}$ ). This shows the observational consequence of the offset center of symmetry of the disk model. Because the particles at the inner edge of the disk (those that contribute most to the disk's brightness) are closer to the star in the forced pericenter direction, $\tilde{\omega}_{f}$, than those in the forced apocenter direction (Fig. (1), they are hotter and so contribute more to the disk's thermal emission than those at the forced apocenter. This is the "pericenter glow" phenomenon, which leads to the horseshoe-shaped highest contour line (the filled-in $1.02 \mathrm{mJy} /$ pixel line), which is pointed in the $\tilde{\omega}_{f}$ direction. This asymmetry is a consequence of $a_{\min }$ and $e_{f}$ only, and its magnitude is determined by $e_{f}$ only. In particular, if there is a gradient of $e_{f}$ across the disk, then it is $e_{f}$ at the inner edge of the disk that controls the magnitude of the asymmetry. The outermost contour plotted on Fig. 7a, which is an offset circle with a radius of $95 \mathrm{AU}$, is that corresponding to $0.17 \mathrm{mJy} /$ pixel. Thus, there is little emission from the outer edge of the disk, justifying the arbitrary use of $a_{\max }=130$ AU in the modeling.

\subsection{Disk Model Orientation}

The two variables that define the orientation of the HR 4796 disk to our line of sight are $\tilde{\omega}_{f}$ and $I_{o b s}$; how they define this orientation is best explained using Fig. 7a. Imagine that the disk starts face-on with the forced pericenter direction pointing to the left. It is then rotated clockwise by $\tilde{\omega}_{f}$ (this is shown in Fig. a. where $\tilde{\omega}_{f}=26^{\circ}$ ), and then tilted by $90^{\circ}-I_{o b s}$ about the dotted line on Fig. 目a. The direction of this tilt, whether the top or bottom of the disk ends up closer to the observer, is not constrained in the modeling, since no account was made for either the extinction of the disk's emission by the disk itself, or for the disk's scattered light (e.g., an observer would see forward-scattered starlight from the closest part of the disk and back-scattered starlight from the farthest part, a phenomenon that could produce an apparent asymmetry in a symmetric disk, Kalas \& Jewitt 1995). If the resulting inclination of the disk's symmetry plane to our line of sight, $I_{o b s}$, is small, then the resulting nearly edge-on observation shows two lobes, one

either side of the star. Since the hotter, brighter, pericenter glow material is predominantly in one of the lobes (unless $\tilde{\omega}_{f}=90^{\circ}$ ), the lobes have asymmetric brightnesses. 


\subsection{Modeling Process and Results}

Pseudo-observations of disk models in the IHW18 and N wavebands were produced that mimicked the real OSCIR (the University of Florida mid-IR imager) observations in both pixel size, 1 pixel $=0$ '.0616 $=4.133$ AU at $67 \mathrm{pc}$ (Jura et al. 1998), and smoothing, using the observed PSFs (which are asymmetric and slightly fatter than diffraction limited, T99), and including the post-observational gaussian smoothing of FWHM $=3$ pixels. The model variables: $a_{\min }, \gamma, I_{o b s}$, $e_{f}, \tilde{\omega}_{f}$, and $\sigma_{t o t}$, were optimized so that the modeled IHW18 observation correctly predicts the observed IHW18 brightness distribution; at the same time, the variable, $D_{t y p}$, was optimized so that the modeled $\mathrm{N}$ band observation correctly predicts the observed $\mathrm{N}$ band brightness.

The model observations were compared with the real observations using the following diagnostics: the lobe brightnesses, $F_{n e, s w}$, and their projected radial offsets from HR 4796A, $R_{n e, s w}$, that were found by fitting a quintic polynomial surface to a $10 \times 10$ pixel region around each of the lobes with 0.1 pixel resolution (the location of HR 4796A in the real observations was also found in this way); and line-cuts through the disk both parallel (e.g., Figs. 8d and 9), and perpendicular (e.g., Figs. 8c and Be) to the line joining the two lobes in the IHW18 observations. An understanding of how the different model variables affect the different diagnostics allowed the modeling process to be decoupled into solving for: the disk's symmetrical structure, defined by $I_{o b s}, a_{m i n}$, and $\gamma$; the particle size $D_{t y p}$; and the disk's asymmetrical structure, defined by $e_{f}$ and $\tilde{\omega}_{f}$. Throughout the modeling, the amount of material in a model, $\sigma_{t o t}$, was scaled so that the model observation predicted the correct observed mean brightness of the lobes in the IHW18

waveband, $F_{\text {mean }}=\left(F_{n e}+F_{s w}\right) / 2=1.40 \pm 0.02 \mathrm{mJy} /$ pixel; its final value, $\sigma_{\text {tot }}=2.03 \times 10^{24} \mathrm{~m}^{2}$, was calculated once the other variables had been constrained.

\subsubsection{Symmetrical Disk Structure}

Since $e_{f}$ and $\tilde{\omega}_{f}$ only pertain to the disk's asymmetrical structure, then for a given $D_{t y p}$, the variables pertaining to the disk's symmetrical structure, $I_{o b s}, a_{\text {min }}$, and $\gamma$, could be solved using a model with $e_{f}=0$; such a model is axisymmetric, and so the variable $\tilde{\omega}_{f}$ is redundant. The inclination of the disk's plane of symmetry to the line of sight, $I_{o b s}=13 \pm 1^{\circ}$, was constrained to give the best fit to the line-cuts perpendicular to the lobes (Figs. Bc and Be); this is in agreement with that found by previous models of HR 4796 disk observations (Koerner et al. 1998; Schneider et al. 1999). Since our model is that of a "fat" disk, a different proper inclination distribution would lead to a different $I_{o b s}$; e.g., if the disk is actually thinner than modeled here, $\left\langle I_{p}\right\rangle<10.2^{\circ}$, then the inferred $I_{o b s}$ is an underestimate, and vice versa. The inner edge of the disk, $a_{\min }=62 \pm 2 \mathrm{AU}$, was constrained such that the model observation reproduces the observed mean radial offset of the lobes from HR $4796 \mathrm{~A}, R_{\text {mean }}=\left(R_{n e}+R_{s w}\right) / 2=58.1 \pm 1.3 \mathrm{AU}$. The semimajor axis distribution, $\gamma=-2 \pm 1$, was constrained to give the best fit to the cut along the line joining the two lobes (Fig. 8d). 


\subsubsection{Particle Size}

Since $D_{\text {typ }}$ was already estimated to be about 2-3 $\mu \mathrm{m}(\$ .2 .2)$, the modeling of the disk's symmetrical structure was repeated for $D_{t y p}=2,2.5$, and $3 \mu \mathrm{m}$. Adjusting $D_{t y p}$ by such a small amount did not affect the inferred symmetrical structure parameters. This was expected, since the $P(18.2 \mu \mathrm{m}, r)$ for each of these $D_{t y p}$ are very similar (Fig. $5 \mathrm{~d}$ ). Remembering that the model is always normalized to predict the observed mean IHW18 lobe brightnesses, the predicted N band lobe brightnesses are compared for the three values of $D_{t y p}$ in Fig. 9. This shows that the particle size can be constrained to be $D_{t y p}=2.5 \pm 0.5 \mu \mathrm{m}$; i.e., the crude method of calculating the particle size of $\$ 5.2 .2$ gives a very good estimate of this size. This particle size means that the total mass of emitting particles in the disk model is $\sim 1.4 \times 10^{-3} M_{\oplus}$, where $M_{\oplus}=3 \times 10^{-6} M_{\odot}$ is the mass of the Earth. However, this is not a useful constraint on the disk's mass, since the disk's mass is expected to be concentrated in its largest particles. The best estimate of the mass of the HR 4796 disk, from submillimeter observations, is that it is between $0.1 M_{\oplus}$ and $1.0 M_{\oplus}$ Jura et al. 1995; Jura et al. 1998), which, as expected, is well above the mass of our disk model.

\subsubsection{Asymmetrical Disk Structure}

The disk's observed asymmetries are defined by: the lobe brightness asymmetry, $\left(F_{n e}-F_{s w}\right) / F_{\text {mean }}=5.1 \pm 3.2 \%$, and the radial offset asymmetry, $\left(R_{s w}-R_{n e}\right) / R_{\text {mean }}=6.4 \pm 4.6 \%$. These asymmetries are also apparent in the disk model, and their magnitudes are determined by both $e_{f}$ and $\tilde{\omega}_{f}$. The lobe brightness asymmetry was used to constrain $e_{f}$ and $\tilde{\omega}_{f}$, and it was found that the $e_{f}$ necessary to cause the $5.1 \pm 3.2 \%$ asymmetry depends on the geometry of the observation according to the relation shown in Fig. $7 \mathrm{~b}$. Thus, for the majority of the geometries, a forced eccentricity of between 0.02 and 0.03 is sufficient to cause the observed brightness asymmetry. In the context of this modeling, the observed brightness asymmetry implies a radial offset asymmetry of $\sim 5 \%$, which is within the limits of the observation. Our final model shown in Figs. 田and 019 assumes a modest value of $e_{f}=0.02$, which corresponds to an $\tilde{\omega}_{f}$ orientation of $26^{\circ}$ (Fig. $\overline{7} \mathrm{~b}$ ). Whether this rotation puts the pericenter glow material above or below the horizontal, $\tilde{\omega}_{f}=26^{\circ}$ or $-26^{\circ}$, is not constrained here, since it has a minimal effect on the observation: in the model observation of Fig. 8, the top of the disk is brighter than the bottom of the disk by a fraction that would be undetectable in the observation due to noise and the disk's unknown residual structure. The line-cuts of Fig. 8 show how well the model fits all aspects of the observation the vertical structure, the horizontal structure, and the lobe location and asymmetry.

\subsubsection{Statistical Significance}

The standard deviations of the OSCIR lobe observations quoted in this section were found using model observations that mimicked the noise present in the OSCIR observations. The 
background sky noise in the IHW18 observation was found to be approximately gaussian with zero mean and a $1 \sigma$ noise per pixel of $0.15 \mathrm{mJy}$ (T99); this was included after the PSF smoothing, but before the post-observational smoothing. Observations of the model were repeated for 50,000 different noise fields to obtain the quoted standard deviations. Since the observed PSF was asymmetric (T99), this introduces an apparent lobe asymmetry of $-0.8 \%$ in an observation of a symmetric disk (one with $e_{f}=0$ ), and so the observed lobe asymmetry is $5.9 \pm 3.2 \%$ from the mean, and its statistical significance is $1.8 \sigma$. While this is small, it does show that the pericenter glow phenomenon is observable with current technology: HR 4796 was observed with the infrared imager OSCIR for only one hour on Keck II (T99); in the background-limited regime, the significance level of any asymmetry increases at a rate $\propto \sqrt{t}$; thus, one good night on a 10 meter telescope should be enough to get a definitive observation of the HR 4796 lobe asymmetry. The real significance of the HR 4796 asymmetry may also be higher than quoted above, since it also seems to be apparent in other observations of this disk (Koerner et al. 1998; Schneider et al. 1999). Even if subsequent observations happen to disprove the existence of the asymmetry, this is still significant, since, as we show in $\$ 6.2$, an asymmetry is to be expected if the companion star, HR 4796B, is on an eccentric orbit.

\section{Interpretation of the HR 4796 Disk Model}

The interpretation of our HR 4796 disk model is broken down into sections that cover discussions of: the dynamics of the disk particles, $\$ 6.1$; the lobe asymmetry, $\$ 6.2$; the emitting particle category, $\$ 6.3$; the origin of the inner hole, $\$ 6.4$; and the residual structure of the observation once the model has been subtracted, $\$ 6.5$.

\subsection{The Dynamic HR 4796 Disk}

Our interpretation of the observed structure of the HR 4796 disk starts with a discussion of the dynamics of the particles in the disk, and where the emitting particles fit into our understanding of the dynamic disk $(\oint 3)$.

\subsubsection{Radiation Forces, $\beta$}

The radiation forces, defined by $\beta$ (eq. [17]), acting on particles in the HR 4796 disk can be found from their optical properties. Fig. $6 \mathrm{~b}$ shows the $\beta$ of particles with the optical properties

assumed in our model ( $(5.2 .1$ ). Thus, particles in the disk with $D<8 \mu \mathrm{m}$ are $\beta$ meteoroids (even the submicron particles have $\beta>0.5$ ), and a good approximation for the non- $\beta$ meteoroids is:

$$
\beta \approx 4 / D
$$


where $D$ is measured in $\mu \mathrm{m}$ (in agreement with eq. 18]). Fig. 9 shows that the emitting particles have $D_{\text {typ }} \approx 2.5 \mu \mathrm{m}$; these particles have $\beta=1.7$. So, the modeling implies that the emitting particles are $\beta$ meteoroids, i.e., that they are blown out of the system on hyperbolic orbits. Since the lifetime of $\beta$ meteoroids is $O(370$ years $)$ for those created at $\sim 70 \mathrm{AU}$, which is much shorter than the age of the HR 4796 system, any $\beta$ meteoroids that are currently in the disk cannot be primordial particles, rather they must be continuously created from a reservoir of larger particles; i.e., the existence of $\beta$ meteoroids implies the existence of a dynamically stable population of larger particles.

If the emitting particles are on hyperbolic orbits, we can use the mass of the disk's emitting particles, $\sim 1.4 \times 10^{-3} M_{\oplus}$, and the emitting lifetime of these particles, $\sim 370$ years, to estimate the mass loss rate of the disk to be $\sim 4 \times 10^{-6} M_{\oplus} /$ year. If this mass loss rate has been sustained over the age of the system, the original disk must have been $\sim 40 M_{\oplus}$ more massive than it is today. In fact, a more massive disk would have had a higher mass loss rate, since this rate increases proportionally with the total cross-sectional area in the disk (i.e., $\propto m_{t o t}^{2 / 3}$ ). This means that if the current disk has a mass $1.0 M_{\oplus}$, the original disk must have had a mass $\sim 7 \times 10^{4} M_{\oplus}$; i.e., the HR 4796 disk may provide evidence for the type of collisional mass loss that may have happened in the early Kuiper belt (Stern \& Colwell 1997).

\subsubsection{Collisional Processes}

The collisional lifetime of the disk's emitting particles can be calculated directly from the disk model using equations (A7) and (A12): $t_{\text {coll }}\left(D_{\text {typ }}\right)<10^{4}$ years across most of the disk (55-85 $\mathrm{AU})$, with a minimum at $\sim 70 \mathrm{AU}$ of $\sim 4500$ years. This collisional lifetime is much less than the age of the HR 4796 system. Thus, the emitting particles cannot be primordial particles (irrespective of whether they are $\beta$ meteoroids). The collisional lifetime of the emitting particles can be corroborated using equation (16). The model's effective optical depth (eq. A88) is its surface density, plotted in Fig. 4, multiplied by the cross-sectional area of a particle in the model, $\sigma=\pi D_{\text {typ }}^{2} / 4$. This peaks at $70 \mathrm{AU}$, where $\tau_{\text {eff }}(70 \mathrm{AU})=5 \times 10^{-3}$, and $t_{\text {per }}=O(370$ years $)$, giving a collisional lifetime that has a minimum of $t_{\text {coll }}\left(D_{\text {typ }}\right) \approx 6000$ years. The disk's effective optical depth at $70 \mathrm{AU}, \tau_{\text {eff }}(70 \mathrm{AU})$, can also be corroborated directly from the observation using equation (A10). The observed edge-on, smoothed lobe brightness is $\sim 1.40 \mathrm{mJy} /$ pixel; this can be scaled to the unsmoothed face-on brightness by the factor 1.10/1.40 (see Figs. Ga and $8 \mathrm{~b})$, which, since each pixel subtends $(0.0616 * \pi / 648000)^{2}$ sr, gives a brightness of $F_{\nu}(18.2$ $\mu \mathrm{m}, 70 \mathrm{AU}) / \Omega_{o b s}=12 \times 10^{9} \mathrm{Jy} / \mathrm{sr}$. For $2.5 \mu \mathrm{m}$ particles, Figs. 5c and fid give $P(18.2 \mu \mathrm{m}, 70$ $\mathrm{AU}) \approx 2.2 \times 10^{12} \mathrm{Jy} / \mathrm{sr}$, thus confirming that $\tau_{\text {eff }}(70 \mathrm{AU}) \approx 5 \times 10^{-3}$ (this is also in agreement with $\tau \approx 5 \times 10^{-3}$ found by Jura et al. 1995). In fact, assuming that the disk's total IHW18 flux density, 857 mJy (T99), comes from particles between $70 \pm 15 \mathrm{AU}$, the unsmoothed face-on brightness of the disk can also be corroborated; equation (A11) with $R_{\star}=67 \mathrm{pc}$ gives $F_{\nu}(18.2$ $\mu \mathrm{m}, 70 \mathrm{AU}) / \Omega_{\text {obs }}=O\left(10^{10} \mathrm{Jy} / \mathrm{sr}\right)$. 
Since particles are only broken up by collisions with particles that have diameters more than a tenth of their own (eq. A2]), the collisional lifetime of the disk's large particles must be longer than that of the smaller emitting particles. Assuming the cross-sectional area distribution to follow equation (15) with $q=11 / 6$ down to particles of size $D_{t y p}$, the collisional lifetime of particles with $D \gg D_{\text {typ }}$ can be estimated as (eq. A15):

$$
t_{\text {coll }} \approx 4 \times 10^{7} \sqrt{D}
$$

where $t_{\text {coll }}$ is measured in years, and D in $\mathrm{km}$. Since particles for which $t_{\text {coll }}<t_{\text {sys }}$ cannot be primordial, this implies that particles currently in the HR 4796 disk that are smaller than 60 $\mathrm{m}$ cannot be original, rather they must have been created in the break-up of larger particles; i.e., these disk particles form a collisional cascade, through which the spatial distributions of the smaller particles are related to that of the larger particles. The disk's particles that are larger than $60 \mathrm{~m}$ are its primordial particles.

\subsubsection{P-R Drag}

The disk's high effective optical depth means that $\beta_{p r}=132$ at $70 \mathrm{AU}$ (eq. [26]), which in turn means that there is not expected to be significant P-R drag evolution for any of the disk's particles. Analysis of the P-R drag evolution of disk particles (eq. 25]) shows that even the most affected particles, those with $\beta=0.5$, have a P-R drag lifetime of $t_{p r}=O(1.6 \mathrm{Myr})$ at $70 \mathrm{AU}$, and that these particles would only make it to $\sim 69.8$ AU before they are broken up by collisions and blown out of the system by radiation pressure. Appendix B, which gives a discussion of lobe asymmetries in disks for which P-R drag is important, gives an analysis that shows that the cumulative effect of $\mathrm{P}-\mathrm{R}$ drag on the orbits of all of a disk particle's ancestors is also insignificant.

\subsubsection{The Dynamic HR 4796 Disk}

Since P-R drag is not an important process in the HR 4796 disk's evolution, there are just three categories of particles in the disk: large particles, $\beta$ critical particles, and $\beta$ meteoroids. If we are to believe the modeled emitting particle size (discussed further in $\S 6.3$ ), the particles that are seen in both the IHW18 and the N band observations are the disk's $\beta$ meteoroids. Since we modeled the disk as if the emitting particles are large particles, this inconsistency needs to be borne in mind in our interpretation of the model.

While the modeling used a distribution of orbital elements that is only appropriate for the disk's large particles, it was the spatial distribution of the disk's emitting material, $\sigma(r, \theta, \phi)$, that was constrained by the modeling, not the distribution of orbital elements, $\sigma(a, e, I, \Omega, \tilde{\omega})$. Therefore, the inferred distribution, $\sigma(r, \theta, \phi)$, which is that shown in Fig. 4 , is an accurate description of the spatial distribution of the disk's emitting particles, whatever their size. Indeed, 
it is in excellent agreement with that inferred from other observations of the HR 4796 disk (Jayawardhana et al. 1998; Koerner et al. 1998; Schneider et al. 1999). If the emitting particles are the disk's large particles, then the inferred model variables have physical interpretations for the distribution of the orbits of the disk's large particles. If, as appears to be the case, the emitting particles are $\beta$ meteoroids, then further modeling of $\sigma(r, \theta, \phi)$ needs to be done to infer the distribution of the orbits of these particles.

However, since the disk's $\beta$ critical and $\beta$ meteoroid particles are created from its large particles, the spatial distributions of all of these particles share a great deal in common (see $\S \S 3.2$ and 4.2): they all have the same plane of symmetry, the same flaring, and the same offset and warp asymmetries, but the radial distributions of the smaller particles are more extended than that of the large particles. This means that if we are seeing the disk's $\beta$ meteoroids, then the spatial distribution of its large particles has a plane of symmetry that is defined by $I_{o b s}$, and an inner edge that is at the same radial location, and that is offset by the same amount and in the same direction, as that shown in Fig. 击 their radial distribution, however, would not be as extended as that of Fig. 4 . Thus, the model parameters $a_{m i n}, e_{f}$, and $\tilde{\omega}_{f}$ have physical interpretations for the distribution of the orbits of the disk's large particles, irrespective of the size of the emitting particles.

\subsection{Interpretation of Lobe Asymmetry: HR 4796's Secular Perturbations}

The lobe asymmetry in the model of $\$$ 河 is due solely to the offset inner edge of the disk. The model shows that secular perturbations amounting to a comparatively small forced eccentricity,

$e_{f}=0.02$, imposed on the orbits of large particles at the inner edge of the disk, $a=62 \mathrm{AU}$, would cause the disk's inner edge to be offset by a sufficient amount to cause the observed $5 \%$ lobe asymmetry. This section considers what kind of a perturber system would impose such a forced eccentricity on the inner edge of the disk, and whether such a system is physically realistic. A discussion of the system's secular perturbations also allows interpretation of the disk's inferred orientation, defined by the parameters $\tilde{\omega}_{f}$ and $I_{o b s}$.

If HR 4796A's binary companion, HR 4796B, is on an eccentric orbit, it would have imposed a forced eccentricity on the disk particles. However, a forced eccentricity could also have been imposed on the disk by an unseen planet close to the inner edge of the disk, a planet which could be responsible for clearing the inner region (e.g., Roques et al. 1994). The secular perturbations imposed on the HR 4796 disk by a perturber system that includes HR 4796B and a putative planet located at the inner edge of the disk are shown in Fig. 10 for the four cases: $M_{p l}=0$, $M_{p l}=0.1 M_{J}, M_{p l}=10 M_{J}$, and just planet. The parameters of the two perturbers are assumed to be:

HR 4796B $M_{B}=0.38 M_{\odot}$ (Jayawardhana et al. 1998); the orbit of HR 4796B is unknown at present (Jura et al. 1993), so the semimajor axis of its orbit is arbitrarily taken as its 
projected distance, $a_{B}=517 \mathrm{AU}$ (Jura et al. 1998; note that we are not assuming that this is the semimajor axis of the ellipse that the star's orbit traces on the sky) - this gives an orbital period of $\sim 7000$ years (eq. [12]), and a timescale for secular perturbations from HR $4796 \mathrm{~B}$ to have built up at $62 \mathrm{AU}$ of $O(1 \mathrm{Myr})$ (eq. [42]); $e_{B}=0.13$, the eccentricity necessary to cause $e_{f}=0.02$ at $a=62 \mathrm{AU}$ if there were no unseen perturbers (eq. 41a]); $I_{B}=0^{\circ}$, defining the reference plane for the analysis.

Planet $M_{p l}$ is a variable measured in Jupiter masses, where $M_{J}=10^{-3} M_{\odot}$ (current observations have limited the size of a planet in the system to $M_{p l}<20 M_{J}$, Jura et al. 1998); $a_{p l}=47$ $\mathrm{AU}$ (see $\$[.4) ; e_{p l}=0.023$, the eccentricity necessary to cause $e_{f}=0.02$ at $a=62 \mathrm{AU}$ if the planet was the only perturber (eq. [41a]); $I_{p l}=5^{\circ}$, an arbitrary choice that represents the fact that the orbital plane of the planet is not necessarily be aligned with that of HR 4796B.

\subsubsection{Just HR 4796B}

For the cases when there is just one perturber in the system, the forced elements in the system can be found from equations (41a) and (41b): the forced eccentricity, $e_{f}$, is determined by the ratio of the semimajor axes of the perturber and the particle, and by the eccentricity of the perturber's orbit, but is independent of the perturber's mass; the forced pericenter, $\tilde{\omega}_{f}$, is aligned with the pericenter of the perturber; and the plane of symmetry of the disk, $y_{f}$, is constant across the disk, and is the orbital plane of the perturber.

So, if the only perturber is HR 4796B, then to impose $e_{f}=0.02$ at $a=62 \mathrm{AU}$, the eccentricity of its orbit would have to be $e_{B}=0.13$; the consequent forced eccentricity imposed on the disk is plotted in Fig. 10a. This also means that if $e_{B}>0.1$, then a brightness asymmetry in this disk of $>5 \%$ would be expected unless adverse geometrical conditions prevented it. The position angle from north of HR $4796 \mathrm{~B}$ relative to HR 4796A is $225^{\circ}$ (Jayawardhana et al. 1998), while that of the SW lobe (i.e., the least bright lobe) is $206^{\circ}$ (T99). For the lobe asymmetry to be the consequence of perturbations from HR 4796B only, HR 4796B must currently be close to its apastron, and its orbital plane must be the plane of symmetry of the disk, i.e., inclined at $13^{\circ}$ to the line of sight. All of these conclusions are consistent with our initial estimate that the semimajor axis of the star's orbit is equal to its observed projected distance, $517 \mathrm{AU}$.

\subsubsection{HR 4796B and a Planet}

Consider the effect of adding a planet at the inner edge of the disk into the HR 4796 systemf If there are two perturbers in the system, then the forced element variation with semimajor

\footnotetext{
${ }^{4}$ The orbital elements of a low-mass planet in the system would, just like the disk particles, have forced and proper components; a high-mass planet would perturb the orbit of HR 4796B.
} 
axis depends both on the masses of the perturbers and on the orientations of their orbits. In the plot of Fig. 10 b,$\tilde{\omega}_{p l}=\tilde{\omega}_{B}+180^{\circ}$ was chosen so that the forced eccentricity (and hence the lobe asymmetry) is aligned with the planet's pericenter for $a<a_{\text {crit }}$, and aligned with HR 4796B's pericenter for $a>a_{\text {crit }}$, where $a_{c r i t}$ is the semimajor axis for which $e_{f}=0$. Since the two perturbers were also chosen to have different orbital planes, a similar change in the disk's alignment is seen in the plot of $I_{f}$ (Fig. 10 d): the disk's plane of symmetry is aligned with the planet's orbital plane at its inner edge, and with the orbital plane of HR 4796B at its outer edge; this could cause an image of the disk to appear warped. Such a warp could be modeled using the same modeling techniques that are described in this paper, and would provide further constraints on the perturbers in the system (even if no warp was observed).

So, it is possible that the brightness asymmetry, and the symmetry plane of the lobes, are determined by a planet close to the edge of the disk that has $M_{p l}>0.1 M_{J}$, rather than by HR 4796B. Using such an analysis, the pericenter glow phenomenon could be used to test for the existence of a planet in the HR 4796 system, but only after the orbit of HR 4796B has been determined; e.g., if $\tilde{\omega}_{B}, e_{B}$ or the plane of HR 4796B's orbit contradicted the observed asymmetry orientation, brightness asymmetry magnitude, or the plane of symmetry of the lobes, then the existence of a planet at the inner edge of the disk with $M_{p l}>0.1 M_{J}$ could be inferred.

\subsubsection{Just Planet}

A double-lobed disk structure could also be observed in a system with no observable companion. The only possible perturber in such a system is an unseen planet, the secular perturbations of which warrant the same kind of discussion as for the case when HR 4796B was the only perturber $(\oint 6.2 .1)$. Depending on the planet's mass, radial location and eccentricity, it too could give rise to a detectable pericenter glow. The only constraint on the planet's mass is that the disk must be old enough for its secular perturbations to have affected the distribution of orbital elements of the disk particles over the age of the system. Since it takes of the order of one precession timescale to distribute the complex eccentricities of collisional fragments around the circle centered on the forced eccentricity, the constraint on the planet's mass can be approximated as that for which is that the age of the system, $t_{s y s}$, is greater than the secular timescale, $t_{s e c} \propto 1 / M_{p l}$ (eq. [42]); for the HR 4796 system this limit is $M_{p l}>10 M_{\oplus}$. The constraint on the planet's eccentricity is even less stringent than for the binary companion because the planet is closer to the edge of the disk: a planet in the HR 4796 system would only need an eccentricity of

$e_{p l}>0.02$ to produce the observed $5 \%$ lobe asymmetry, and the forced eccentricity imposed on the disk by a planet with $e_{p l}=0.023$ is plotted in Fig. 10 c. So, the signature of even a low-mass planet would not escape detection and symmetrical double-lobed features are unlikely to be observed in systems that contain planets. 


\subsubsection{Other Considerations}

If the disk itself is massive enough to cause significant gravitational perturbations to the orbits of the disk particles, then the mass of the disk should be incorporated into the analysis of the secular perturbations in the system. A massive disk could dampen the eccentricity of a planet at the inner edge of the disk (Ward \& Hahn 1998), thus reducing the offset asymmetry.

\subsubsection{Fomalhaut}

The Fomalhaut disk lobes may have asymmetric brightnesses (Holland et al. 1998), but the statistical significance of this asymmetry is low. Fomalhaut is a wide visual binary system. Gliese 879 is Fomalhaut's common proper motion companion (Barrado Y Navascués et al. 1997); the two stars are separated by $\sim 2^{\circ}$, which corresponds to a projected separation of $O(55,000 \mathrm{AU})$ at $7.7 \mathrm{pc}$. At such a distance, the forced eccentricity imposed on the disk by the binary star is insignificant (eq. 41a). A secular perturbation offset asymmetry in this disk would be expected only if there is a planet in the disk that has a non-circular orbit.

\subsection{Discussion of Emitting Particle Category}

The emitting lifetime of the disk's $\beta$ meteoroids, $O(370$ years), is less than the emitting lifetime of their parents, $O\left(10^{4}\right.$ years $)$; equivalently, $\tau_{\text {eff }}<0.1$. Thus, our understanding of the dynamic disk implies that the disk's cross-sectional area distribution should not contain a significant amount of $\beta$ meteoroids. Rather, since there are no P-R drag affected particles in the disk, the disk's emission is expected to come from its $\beta$ critical particles, and its smallest large particles (33.2.2). Not all disk particles have the same composition and morphology; even if this were a close approximation, there is, as yet, no evidence to suggest whether the particle properties chosen in our modeling ( $\$$.2.1) are correct. Are we indeed seeing the disk's $\beta$ meteoroid particles, or did the assumptions of the modeling lead us to this conclusion?

Consider the initial crude estimate of the particle size (\$5.2.2); this proved to be an accurate method for estimating the particle size (\$5.4.2). If different assumptions had been made about the particles' properties (e.g., if the particles had been assumed to be made of ice) or morphologies (e.g., if the particles had been assumed to be like the "bird's nest" structures of Gustafson 1994), both Figs. 6a and 6b would be different, and different conclusions might have been drawn about the $\beta$ of the emitting particles. If a size distribution had been included in the modeling, this

would also have affected our conclusions. These are considerations that should be modeled before any firm conclusions about the dynamics of the emitting particles can be reached. However, since irrespective of their assumed properties large particles have black body temperatures and brightness ratios similar to those of the $D>100 \mu \mathrm{m}$ particles in Fig. 6 $\mathrm{b}$, a flexible interpretation 
of the observed brightness ratio is that the emitting particles must have temperatures that are hotter than black body. Thus, the emitting particles are either small (e.g., the simple analysis of $\$ 5.2 .1$ implies that $D<10 \mu \mathrm{m}$ ), in which case they are likely to be $\beta$ meteoroids (e.g., 10 $\mu \mathrm{m}$ particles have $\beta>0.5$ unless they have densities $>3000 \mathrm{~kg} / \mathrm{m}^{3}$, eq. [18]), or they are large particles that are made up of smaller hotter particles.

The assumptions about the particle properties in the model could also have affected our conclusions about the collisional lifetime of the emitting particles. Consider the estimate of $\tau_{\text {eff }}(70$ AU) derived from the IHW18 lobe brightness $(\$ 6.1 .2)$. Changing the properties of particles in the model would change the estimate of $\tau_{\text {eff }}$ because of the resultant changes in $P(18.2 \mu \mathrm{m}, r)$; e.g., if we had modeled the disk using 30-50 $\mu$ m particles, we would have had to put more cross-sectional area in the model for it to give the observed lobe brightness. Fig. 5c shows that for astronomical silicate Mie spheres that are larger than $0.01 \mu \mathrm{m}$ in diameter, $P(18.2 \mu \mathrm{m}, 70 \mathrm{AU})$ must lie in the range $0.034-2.3 \times 10^{12} \mathrm{Jy} / \mathrm{sr}$, depending on whether the disk's cross-sectional area is concentrated in its 30-50 $\mu \mathrm{m}$ particles that emit at cool temperatures, or in its 2-3 $\mu \mathrm{m}$ particles that are small and hot, but large enough to emit efficiently at $18.2 \mu \mathrm{m}$. For particles with different optical properties, $P(18.2 \mu \mathrm{m}, 70 \mathrm{AU})$ could be below $0.034 \times 10^{12} \mathrm{Jy} / \mathrm{sr}$ if the particles have temperatures well below black body (or if they have low emission efficiencies); equally, it could be higher than $2.3 \times 10^{12} \mathrm{Jy} / \mathrm{sr}$ if the particles are hotter than the $2-3 \mu \mathrm{m}$ astronomical silicate Mie spheres. Taking $0.034 \times 10^{12} \mathrm{Jy} / \mathrm{sr}$ as a lower limit for $P(18.2 \mu \mathrm{m}, 70 \mathrm{AU})$ implies that $\tau_{\text {eff }}(70 \mathrm{AU})<0.35$, giving a collisional lifetime for the emitting particles that could be as low as 85 years. However, given the temperature of the emitting particles inferred from the observed brightness ratio, $O\left(10^{4}\right.$ years) remains the best estimate for their collisional lifetime. A further note of caution is necessary about the inferred collisional lifetime: Appendix A assumes collisions between disk particles to be either catastrophic or irrelevant. While this may be appropriate for the disk's larger particles, since these are likely to be solid bodies, collisions between its smaller particles, which may have fluffy "bird's nest" structures (Gustafson 1994), could be more erosive than destructive, and could lead to significant grain growth.

In conclusion, neither observational, nor theoretical considerations can provide a definitive answer as to the dynamics of the emitting particles. However, the confirmation of the emitting particles' collisional lifetimes means that we can be sure that these particles are not primordial, and that there are no P-R drag affected particles in the disk. $\beta$ meteoroids remain the most likely candidate for the emitting particles. Mid-IR emitting particles that are on hyperbolic orbits have been inferred from observations of the disks around both $\beta$ Pictoris (Telesco et al. 1988) and HD 141569 (Fisher et al. 1999).

\subsection{Origin of the Inner Hole}

Whatever the size of the emitting particles, analysis of the optical depth of the disk's inner region (T99) shows that it is a few hundred times less than that of the outer disk, and so there 
are very few emitting particles in this region. Because this central hole is necessary for the secular perturbation offset asymmetry to be observed (without the hole only the radial offset could be observed), its physical origin requires attention. Since the existence of small emitting particles in the disk requires the existence of large particles, the question to answer is why there are so many large particles in the outer disk, but so few in the inner disk? Either the physical conditions were such that they were able to form in the outer region, but not in the inner region, or they formed across the whole disk, but those formed in the inner region have since been removed. Rather than discussing the planetary formation process and the stage of the system's evolution (although these are of utmost importance in determining the physics of the disk), this section offers possible dynamical explanations for the removal of the large particles.

If a planet (or planets) exists interior to the inner edge of the disk, then resonance overlap removes all material from a region of radial width $\sim 1.3 a_{p l}\left(M_{p l} / M_{\star}\right)^{2 / 7}$ about the planet's orbit within about 1000 orbital periods (Wisdom 1980; Duncan, Quinn, \& Tremaine 1989). Material is also removed from the secular resonance regions (Lecar \& Franklin 1997) - the origin of these regions, which cover the range of semimajor axes on the plots of Figs. 10 b and 10d where $e_{f}, I_{f} \rightarrow \infty$, was discussed in $\S 4.1 .2$. The radial distribution of material would also be affected by planetary radial migration (Malhotra 1995; Trilling et al. 1998). However, such mechanisms can only explain the total lack of large particles in the inner region by invoking a system with either many planets, or just one planet that is either very large, or on a very eccentric orbit. Since all of these mechanisms take longer than a particle's orbital period to take effect, they would cause an inner cut-off in the disk particles' semimajor axes (as opposed to a cut-off in radial distance from the star).

To estimate the orbit of a putative planet at the inner edge of the HR 4796 disk that is causing the cut-off, consider the inner edge of the Kuiper belt. There is almost no Kuiper belt material on orbits with semimajor axes interior to that of Pluto (Jewitt 1999), which is in 2:3 resonance with Neptune. This is supposed to be the result of resonance sweeping that occurred as Neptune's orbit expanded early in the history of the solar system due to the clearing of planetesimal debris from the inner solar system and the formation of the Oort cloud (Malhotra 1995). By analogy, assuming that the inner cut-off of the disk's large particles occurs at the planet's $2: 3$ resonance location, and that this cut-off can be described by $a_{\text {min }}=62 \mathrm{AU}$, we can estimate the orbit of the planet to have a semimajor axis of $a_{p l}=a_{m i n}[\mathbf{2} / \mathbf{3}]^{2 / 3}=47 \mathrm{AU}$, giving an orbital period of $\sim 200$ years (eq. [12]).

\subsection{Interpretation of the Residual Structure}

So far, no explanation has been offered for the structure of the residuals (what is left after subtracting the model from the observation, see T99). Analogy with the zodiacal cloud implies that there could be a population of warmer dust in the inner region that may be unrelated to the dust in the outer disk. Depending on the perturbers in the inner region, such dust could contain 
considerable structure. Analysis of emission from such regions would reveal a great deal about the system's perturbers, however, this would not be easy, since the resolution required to map such small-scale structure is at the limit of current technological capabilities. In addition, such emission is masked by that of both the stellar photosphere and the outer disk, the accurate subtractions of which are difficult.

There may also be residual structure associated with the outer regions of the disk. If there is a planet orbiting HR 4796A close to the inner edge of the disk, then the distribution of large particles in the outer disk would contain structure associated with the planet's gravitational perturbations in addition to the secular perturbations already discussed (Dermott et al. 1994; Malhotra 1996; Dermott et al. 1998; Ward \& Hahn 1998). Some of the emitting particles might be trapped in its resonant ring. Such a ring could be responsible for some of the observed lobe asymmetry. The existence of such a ring would give the inner edge of the disk structure that co-orbits with the planet; i.e., observations of this structure would vary on timescales of $\sim 200$ years, offering a method of distinguishing between this structure and the large-scale background structure, which would vary on secular timescales of $O(1 \mathrm{Myr})$. The asymmetric structure of the Earth's resonant ring includes a $0.2^{3} \mathrm{AU}^{3}$ cloud of dust located permanently in the Earth's wake with a number density $\sim 10 \%$ above the background (Dermott et al. 1994). Observations of such an asymmetric structure in an exosolar disk could be modeled using the same techniques that were used to model the Earth's resonant ring (Dermott et al. 1994; Jayaraman \& Dermott 1999), possibly allowing us to determine the presence, location, and even the mass of the perturbing planet (Dermott et al. 1998). However, the evidence suggests that such observations may not be possible with currently available technology. In fact, calculations show that when viewed from a distant point in space normal to the ecliptic plane, the Earth's "wake" would only have an IR signal $O(0.1)$ times that of the Earth (Backman 1998). So, regardless of the resolution requirements, if one were to observe the solar system from outside, it would be easier to detect the Earth directly than to infer its existence from the structure of the zodiacal cloud.

The Earth's resonant ring is a result of the trapping of particles that are evolving into the inner solar system due to P-R drag. Another method of forming a resonant ring is for a planet to undergo radial migration of its orbit, trapping all particles exterior to its orbit into its strongest resonances; such an interaction is supposed to have happened between Neptune and the Kuiper belt (Malhotra 1995). The amount of disk material that is trapped in a Kuiper belt ring depends on how much radial migration has taken place to the planets orbit. A ring with all of its particles trapped in the 1:2 and 2:3 resonances with the planet (these are the strongest resonances) would have three lobes, with the planet residing in an "empty" fourth lobe. Could this be the cause of the tri-lobed structure observed in $\epsilon$ Eridani (Greaves et al. 1998)? 


\section{Conclusions}

The primary intent of this paper was to show how the long-term effect of the gravitational perturbations, i.e., the secular perturbations, of a massive perturber could be the cause of the $\sim 5 \%$ brightness asymmetry of the double-lobed feature seen in observations of the HR 4796 disk (T99):

1. We showed how the secular perturbations of a massive perturber in a disk impose a forced eccentricity on the orbits of particles in the disk, thus causing the disk's center of symmetry to be offset from the star towards the perturber's apastron. We also showed how the same perturbations impose a forced inclination on the particles' orbits, which, if there is more than one perturber in the disk, could cause the disk to be warped.

2. We produced a model of the HR 4796 disk that accurately maps the $18.2 \mu \mathrm{m}$ brightness distribution observed by T99; this model is based on a consideration of the dynamics of the particles in the disk. The model shows how the brightness of a disk that has an inner clear region, that also has an offset center of symmetry caused by a forced eccentricity imposed on the disk particles' orbits, would be asymmetric, since the inner edge of one side of the disk is closer to the star, and so is hotter and brighter, than the other side. We showed that a forced eccentricity as small as 0.02 is all that is necessary to cause a $5 \%$ lobe brightness asymmetry in the HR 4796 disk.

3. If the eccentricity of orbit of the companion star, HR 4796B, is larger than 0.13 , then a forced eccentricity of 0.02 is to be expected. However, if there is a planet of mass $>0.1 M_{J}$ located close to the inner edge of the disk, then the forced eccentricity, and hence the asymmetry, imposed on material in the disk's lobes is controlled by the planet rather than the binary companion; this could also cause the disk to be warped. If a forced eccentricity is indeed the cause of the observed lobe asymmetry then observations that constrain the orbit of HR 4796B would help to clarify whether such a planet exists. If the HR 4796 system had no binary companion, a forced eccentricity of 0.02 could have been imposed on the disk by a lone planet with a mass of $>10 M_{\oplus}$, and an eccentricity of $>0.02$.

4. The statistical significance of the HR 4796 disk's lobe asymmetry in the observations of T99 is only at the $1.8 \sigma$ level, however, it is also apparent in the observations of other authors (Koerner et al. 1998; Schneider et al. 1999). It would take one good night on a 10 meter telescope to get a clear observation of the HR 4796 asymmetry. Thus, the indirect detection of planets, even small planets, hiding in circumstellar disks is clearly within reach using these dynamic modeling techniques. This is particularly important, since the direct detection of planets around even nearby stars is well beyond current capabilities (Backman 1998), and indirect detection techniques such as radial velocity (Marcy \& Butler 1998) or astrometric Gatewood 1996) techniques, permit detection only of very massive planets that are close to the star. 
5. If there is a planet close to the inner edge of the disk, many of the disk's particles could be trapped in resonance with that planet, thus forming a resonant ring. Such a ring would give the inner edge of the disk structure that rotates on the timescale of the orbital period of the planet, $\sim 200$ years. This structure could be contributing to the observed lobe asymmetry, and may also be present in the residuals of the observation. This possibility could be explored with further observations. Resonant rings may be the predominantly observable structures of some exosolar systems, such as that recently observed around $\epsilon$ Eridani (Greaves et al. 1998).

The HR 4796 disk modeling also revealed important information about the large-scale symmetrical structure of the disk, as well as about the dynamic properties of its emitting particles:

7. The spatial distribution of material in the disk inferred from our modeling matches that inferred by other authors (Jayawardhana et al. 1998; Koerner et al. 1998; Schneider et al. 1999). The surface density of cross-sectional area in the disk peaks at $\sim 70$ AU from HR 4796A, falling off $\propto r^{-3}$ outside this radius, dropping by a factor of $\sim 2$ between 70 and 60 $\mathrm{AU}$, and falling to zero by $45 \mathrm{AU}$. This soft inner edge to the disk is to be expected if the disk particles' orbits are eccentric.

8. Assuming the particles to be astronomical silicate Mie spheres, the diameter of the emitting particles was estimated to be $D_{t y p}=2-3 \mu \mathrm{m}$. Particles this small have radiation forces that are characterized by $\beta \approx 2$, and so are blown out of the system on hyperbolic orbits on timescales of $\sim 370$ years. The HR 4796 disk is very dense; the collisional lifetime of its emitting particles is $\sim 10^{4}$ years. Thus, the emitting particles cannot be primordial particles, rather they must be continuously created from a reservoir of larger particles. The collisional lifetimes of all of the disk's particles are shorter than their P-R drag lifetimes; i.e., none of the disk's particles are affected by P-R drag. Further investigation of the particles' properties needs to be done before any firm conclusions can be reached about whether the disk is dense enough to support a population of particles on hyperbolic orbits that is large enough to dominate the disk's emission. If the emitting particles are on hyperbolic orbits, the modeling implies a mass loss rate for the disk $\sim 4 \times 10^{-6} M_{\oplus} /$ year, and further modeling would have to be done to ascertain the spatial distribution of the dynamically stable population of large particles from which these emitting particles originated.

\section{A. Collisional Lifetime of Disk Particles}

Consider a collision between two disk particles, the larger of which is denoted by the subscript 1 , and the smaller by the subscript 2. For this collision to be "catastrophic", that is, for it to result in the break-up of the larger particle, the impact energy of the collision must be large enough both to overcome the tensile strength of the larger particle, and to impart enough energy 
to the collisional fragments to overcome its gravitational binding energy. In the asteroid belt this limit means that a collision is only catastrophic if $m_{2} / m_{1} \geq 10^{-4}$ (Dohnanyi 1969). Since the impact energy of a collision is $\propto m_{2} v_{\text {rel }}^{2}$, assuming that exosolar disk particles have similar tensile strengths to the solar system's asteroids, this limit can be scaled to exosolar disks by the square of the ratio of the mean relative velocity of collisions in the asteroid belt (at $\sim 3 \mathrm{AU}$ ), $v_{r e l} \approx 5 \mathrm{~km} / \mathrm{s}$ (Vedder 1998), to that of collisions in the exosolar disk. The mean relative velocity of collisions in exosolar disks can be described by:

$$
v_{r e l}(r)=f(e, I) v(r)
$$

where $f(e, I)$ is some function of the disk particles' eccentricities and inclinations, and $v(r)=30 \sqrt{\left(M_{\star} / M_{\odot}\right)\left(a_{\oplus} / r\right)} \mathrm{km} / \mathrm{s}$ is the average velocity of particles at $r$ (eq. 114 with $a$ replaced by $r$ ). Thus, assuming $f(e, I) \approx 0.3$ as for collisions in the asteroid belt, an exosolar disk particle of diameter $D \propto m^{1 / 3}$, would only suffer a catastrophic collision if the other particle in the collision had a diameter $\geq D_{c c}(D)$, where

$$
D_{c c}(D)=0.03\left[\left(M_{\odot} / M_{\star}\right)\left(r / a_{\oplus}\right)\right]^{1 / 3} D .
$$

The "collisional lifetime", i.e., the mean time between catastrophic collisions, of a particle of diameter $D$, at a location in a disk denoted by $r, \theta$, and $\phi$, is the inverse of its catastrophic collision rate (Kessler 1981):

$$
t_{\text {coll }}(D, r, \theta, \phi)=\left[R_{\text {coll }}(D, r, \theta, \phi)\right]^{-1},
$$

where

$$
R_{\text {coll }}(D, r, \theta, \phi)=\sigma_{c c}(D, r, \theta, \phi) v_{r e l}(r),
$$

$\sigma_{c c}(D, r, \theta, \phi)$ is the catastrophic collision cross-section seen by the particle, and $v_{r e l}(r)$ is the mean encounter velocity of disk particles at $r$ (eq. A1]). Using the definition of a disk's structure given by equation (蛋), this catastrophic collision cross-section is:

$$
\sigma_{c c}(D, r, \theta, \phi)=f_{c c}(D, r) \sigma(r, \theta, \phi)
$$

where

$$
f_{c c}(D, r)=\int_{D_{c c}(D)}^{D_{\max }}\left(1+D / D^{\prime}\right)^{2} \bar{\sigma}\left(D^{\prime}, r\right) d D^{\prime}
$$

and $D_{c c}(D)$ is the smallest particle with which a catastrophic collision could occur (eq. [A2]).

However, unless $t_{\text {coll }} \ll t_{\text {per }}$, the particle's orbit takes it through a range of $\theta$ and $\phi$ before a collision occurs (there is also a variation of $r$ along the particle's orbit due to the eccentricity of its orbit). Thus, it is more appropriate to calculate the particle's collisional lifetime using the mean catastrophic collision rate of the particles in the size range $D \pm d D / 2$ that are in the spherical shell of radius, $r$, and width $d r$. Consider an element of this shell that has a volume, $d V=r^{2} d r d \theta \cos \phi d \phi$. The number of particles in the diameter range $D \pm d D / 2$ in this element is 
given by $n(D, r, \theta, \phi) d D d V$, and each of these particles has a catastrophic collision rate given by equation ( $\mathrm{A} 4)$. Integrating over the whole shell gives:

$$
t_{\text {coll }}(D, r)=\frac{\int_{-I_{\max }}^{+I_{\max }} \int_{0}^{2 \pi} \sigma(r, \theta, \phi) d \theta \cos \phi d \phi}{\int_{-I_{\max }}^{+I_{\max }} \int_{0}^{2 \pi}[\sigma(r, \theta, \phi)]^{2} d \theta \cos \phi d \phi * f_{c c}(D, r) v_{r e l}(r)},
$$

where $I_{\max }$ is the maximum inclination of the disk particles' orbits to the reference plane.

Equation (A7) can be simplified by considering a cylindrical shell, defined by $r, \theta$, and $z$, rather than a spherical one. An element of the cylindrical shell has a volume $d V=r d r d \theta d z$, and the corresponding collisional lifetime of a particle in the shell is given by equation (A7), but with $\phi, \cos \phi d \phi$ and $\pm I_{\max }$, replaced by $z, d z$, and $\pm h$, where $h=r \sin I_{\max }$. Here we introduce the parameter $\tau_{e f f}$, the disk's face-on effective optical depth:

$$
\tau_{e f f}(r)=\int_{-h}^{+h} \sigma(r, \theta, z) d z,
$$

where the dependence on $\theta$ has been dropped since orbits sample the full range of $\theta$. This is not a true optical depth, since that would include a consideration of the particles' extinction coefficients $\left(Q_{e x t}=Q_{a b s}+Q_{s c a}\right)$; rather, it is the disk's face-on surface density of cross-sectional area, which is equal to its true optical depth if its particles had $Q_{\text {ext }}=1$. Assuming that $\sigma(r, \theta, z)$ is independent

of $z$, so that $\int_{-h}^{+h}[\sigma(r, \theta, z)]^{2} d z=0.5 \tau_{e f f}(r)^{2} / h$, and that the encounter speed is determined by the vertical motion of particles in the disk, so that $f(e, I) \approx \sin I_{\max }$, equation (A7) can be simplified to:

$$
t_{\text {coll }}(D, r)=\frac{t_{p e r}(r)}{\pi f_{c c}(D, r) \tau_{e f f}(r)}
$$

where $t_{p e r}(r)$ is the average orbital period of particles at $r$ (eq. [12] with $a$ replaced by $r$ ).

A disk's effective optical depth, $\tau_{\text {eff }}$, can be estimated observationally from equation (6):

$$
\tau_{e f f}(r) \approx\left(F_{\nu}(\lambda, r) / \Omega_{o b s}\right) / P(\lambda, r),
$$

where $F_{\nu} / \Omega_{o b s}$ is the disk's face-on unsmoothed brightness. The disk's face-on unsmoothed brightness can be calculated either from the observed brightness, making corrections to account for both the disk's orientation, as well as for the PSF smoothing, or from the disk's total flux density, $F_{\nu}(\lambda)$, and assuming the disk material to be evenly distributed between $r \pm d r / 2$ :

$$
F_{\nu}(\lambda, r) / \Omega_{o b s}=F_{\nu}(\lambda) C_{f} R_{\star}^{2} / r d r,
$$

where $C_{f}=6.8 \times 10^{9} \mathrm{AU}^{2} / \mathrm{pc}^{2} / \mathrm{sr}$, and $R_{\star}$ is the distance of the star from the observer.

\section{A.1. Collisional Lifetime of Particles with the Most Cross-sectional Area}

Consider the particles in a disk that make up most of the disk's cross-sectional area, i.e., those with diameters close to $D_{t y p}$. By definition, these particles are most likely to collide with 
each other (a collision that would definitely be catastrophic), and so their collisional lifetime can be found from equation (A7) using the approximation:

$$
f_{c c}\left(D_{\text {typ }}, r\right) \approx 4
$$

Applying this approximation to equation (A9) gives equation (16), which is also the collisional lifetime determined by Artymowicz (1997) for particles in $\beta$ Pictoris.

\section{A.2. Collisional Lifetime of a Disk's Large Particles}

The collisional lifetime of particles of different sizes in a disk differ only by the factor $f_{c c}(D, r)$. This factor can be ascertained by making assumptions about the disk particles' size distribution. Assuming that the size distribution of equation (15) holds for disk particles between $D_{\min }$ and $D_{\text {max }}$, the normalized cross-sectional area distribution is given by:

$$
\bar{\sigma}(D)=(3 q-5) D^{4-3 q} / D_{\min }^{5-3 q}
$$

Substituting into equation (A6) gives:

$$
f_{c c}(D)=\left(\frac{X D}{D_{\min }}\right)^{5-3 q}\left[1+\frac{6 q-10}{(3 q-4) X}+\frac{3 q-5}{(3 q-3) X^{2}}\right]
$$

where $X=D_{c c}(D) / D$ for $D_{c c}(D)>D_{\text {min }}$, and $X=D_{\text {min }} / D$ for $D_{c c}(D) \leq D_{\text {min }}$; the collisional lifetime of particles in a disk with this distribution is a minimum for particles for which $D_{c c}(D)=D_{\min }$. The size distribution of particles in a real disk is more complicated than equation (A13), however, equation (A14) can be used to give a crude approximation for the collisional lifetime of a disk's large particles:

$$
t_{\text {coll }}(D, r) \approx t_{\text {coll }}\left(D_{\text {typ }}, r\right) *\left(D_{c c}(D) / D_{\text {typ }}\right)^{3 q-5} .
$$

\section{A.3. Other Considerations for a Particle's Collisional Lifetime}

If the P-R drag lifetime, $t_{p r}$ (eq. 25]), is comparable to, or shorter than $t_{\text {coll }}$, then the effect of P-R drag on the collisional lifetime must be accounted for; e.g., on average, particles from a parent at $r_{\text {parent }}$, survive until they reach $r_{\text {coll }}$ where $\int_{r_{\text {parent }}}^{r_{\text {poll }}} \frac{800\left(M_{\odot} / M_{\star}\right) r}{t_{\text {coll }}(r) \beta} d r=1$. Also, if there is a significant change in $r$ along a particle's orbit due to the eccentricity of its orbit, a similar analysis can be done to take this into account when calculating its collisional lifetime. By considering the washer-like disk of particles on orbits with $a, e$, and random $\tilde{\omega}$ (Sykes 1990): $\left.t_{\text {coll }}(a, e)=\pi\left[\int_{q}^{q^{\prime}}(r / a) /\left[t_{\text {coll }}(r) \sqrt{(r-q)\left(q^{\prime}-r\right.}\right)\right] d r\right]^{-1}$, where $q=a(1-e)$ is this disk's inner edge, and $q^{\prime}=a(1+e)$ is its outer edge. 


\section{B. Lobe Asymmetries in Disks where P-R Drag is Important}

\section{B.1. Offset and Warp of a Disk's P-R Drag Affected Particles}

To find the secular evolution of the orbital elements of a particle that is affected by P-R drag, i.e., one with $\beta_{p r}<\beta<0.1$, the equations governing the evolution of its complex eccentricity, $\dot{z}=\dot{z}_{\text {sec }}-2.5\left(\alpha / a^{2}\right) z$ (eqs. [24] and [37a]), and its complex inclination, $\dot{y}=\dot{y}_{\text {sec }}$ (eq. [37b]), must both be solved in conjunction with the P-R drag evolution of its semimajor axis (eq. 223]). While the solution given by equations (39a) and (39b) is no longer applicable, the decomposition of the particle's complex eccentricity and complex inclination into forced and proper elements, and the physical meaning of these elements, is still valid; however, each of these elements now depends on the particle's dynamical history.

Consider the P-R drag affected particles created by the break-up of the asteroid family group described in $\$ 4.2 .1$. Immediately after they are created, the orbital elements of these particles are the same as those of the rest of the family; i.e., they have semimajor axes $a$, and complex eccentricities and complex inclinations that are uniformly distributed in these planes around circles of radii equal to the proper elements of the family, $e_{p}$ and $I_{p}$. The dynamical evolution of a wave of these particles, i.e., those that were created at the same time, can be followed by numerical integration to ascertain how the orbital elements of the particles in the wave vary as their semimajor axes decrease due to P-R drag; this is the "particles in a circle" method Dermott et al. 1992). It was found that the complex eccentricities and complex inclinations of a wave of particles originating in the asteroid belt remain on circles, and that as the wave's semimajor axis, $a_{\text {wave }}$, decreases: its effective proper eccentricity (the radius of the wave's circle in the complex eccentricity plane) decreases $\propto e_{p} *\left(a_{\text {wave }} / a\right)^{5 / 4}$; its effective proper inclination (the radius of the wave's circle in the complex inclination plane) remains constant at $I_{p}$; the distributions of the particles' $\tilde{\omega}_{p}$ and $\Omega_{p}$ remain random; while its effective forced elements (the centers of the circles in the complex eccentricity and complex inclination planes) have a more complicated variation (Dermott et al. 1992; Liou 1993).

Thus, the orbital element distributions, $n(z)$ and $n(y)$, of P-R drag affected particles are like that of the large particles, in that they are the addition of $z_{f}$ and $y_{f}$ to symmetrical proper element distributions; however, their forced and proper elements are different for particles from different families, as well as being different for particles of different sizes and with different orbital semimajor axes. This means that their spatial distribution is subject to offset and warp secular perturbation asymmetries.

\section{B.2. Origin of Inner Hole}

For disks in which P-R drag is a significant physical process, the existence of an inner hole implies not only that there must be no large particles in the inner region, but also that there 
must be some mechanism that prevents the particles that are in the outer disk from evolving into the inner disk by P-R drag. In HR 4796, and other disks in which P-R drag is insignificant, this mechanism is collisions; i.e., particles created in the outer disk are broken up by collisions before they reach the inner region. This section presents dynamical explanations of how a planet located at the inner edge of the disk would help to prevent the emitting particles from reaching the inner disk.

This is not just important for disks in which its individual particles are not affected significantly by P-R drag in their lifetime, since while the inner edge of a disk's largest particles may be at $a_{\text {min }}$, the cumulative effect of P-R drag on all of the stages of the collisional cascade that a primordial particle goes through before the fragments are small enough to be blown out of the system by radiation pressure could mean that the inner edge of these blow-out particles is further in than $a_{\text {min }}$. To assess whether the cumulative effect of P-R drag has an impact on the inner edge of the emitting particles in the HR 4796 disk, consider its size distribution to follow that assumed in equation (A13), where $D_{\min }=D_{t y p}=2.5 \mu \mathrm{m}$, and $q=11 / 6$. The lifetime of an intermediate particle can be found using equations ( $(\mathrm{A9})$ and ( $\mathrm{A14})$, and the amount of P-R drag evolution in its lifetime is (eqs. 23] and [43]):

$$
d a \approx-0.0125 t_{\text {coll }}(D, r) / D a,
$$

where $d a$ and $a$ are measured in $\mathrm{AU}, D$ in $\mu \mathrm{m}$, and $t_{\text {coll }}(D, r)$ in years. Assuming that the largest fragment created in a collision has a diameter half that of the original particle, a $\beta$ meteoroid particle (e.g., a $2.5 \mu \mathrm{m}$ particle) at $62 \mathrm{AU}$, assuming it to have originated from a gravitationally bound particle (i.e., one with $\beta<0.5$ ), can at the very most be removed by 27 generations from its primordial ancestor, which would at the very most have been originally at $62.1 \mathrm{AU}$.

One consequence of a planet at the inner edge of the disk is that it accretes some of the particles that pass it on their way into the inner region. A simple estimate for the proportion of particles lost in such a way can be obtained by considering the P-R drag evolution of a torus of particles with orbital elements $a, e, I$, and random $\Omega, \tilde{\omega}$ and $\lambda$; the volume of this torus is $V_{\text {tor }}=8 \pi a^{3} e \sin I$ (Sykes 1990). In the time it takes for the torus to pass the planet, $\Delta t=(1602 / \beta)\left(M_{\odot} / M_{\star}\right)\left(a_{p l} / a_{\oplus}\right)^{2} e$ yrs, the planet accretes a volume of dust given by Kessler 1981): $V_{a c c}=\sigma_{c a p} v_{r e l} \Delta t$, where $\sigma_{c a p}=\pi R_{p l}^{2}\left(1+v_{e}^{2} / v_{r e l}^{2}\right)$ is the capture cross-sectional area, $R_{p l}$ is the radius of the planet, $v_{e}=\sqrt{2 G M_{p l} / R_{p l}}$ is the escape velocity of the planet, and $v_{r e l}$ is the mean relative velocity of encounter between the planet and the particles. Thus, the proportion of dust accreted onto the planet, $P=V_{a c c} / V_{t o r}$, is given by:

$$
P \approx \frac{12}{\beta \sqrt{1-\beta}}\left(\frac{M_{\odot}}{M_{\star}}\right)^{3 / 2}\left(\frac{M_{p l}}{M_{\odot}}\right)^{4 / 3}\left(\frac{a_{\oplus}}{a_{p l}}\right)^{1 / 2}\left(\frac{\rho_{J}}{\rho_{p l}}\right)^{1 / 3} g(e, I),
$$

where $\rho_{J}=1330 \mathrm{~kg} / \mathrm{m}^{3}$ is the mean density of Jupiter, $g(e, I)=\left[\left(v_{r e l} / v\right) \sin I\right]^{-1}$, and $v$ is the velocity of the particle; e.g., if $g(e, I)=100$, then a Jupiter-like planet at the inner edge of the disk would need $M_{p l}>30 M_{J}$ to accrete all of the particles passing it (i.e., $P=1$ for $\beta<0.5$ ). 
Another consequence of a planet at the inner edge of the disk is that it traps some of the disk particles into its exterior mean motion resonances. The resulting resonant ring has three consequences that may aid with the formation of a clear inner region. Firstly, the calculation of the probability of accretion onto the planet given in equation (B2) does not apply to the particles that are trapped in the ring. It is thought that resonance trapping helps the accretion process, since trapped particles may leave the resonance upon a close encounter with the planet Kortenkamp \& Dermott 1998). This means that the constraint on the mass of the planet derived in the last paragraph is an upper limit for the accretion process to be the sole removal mechanism. Secondly, the ring increases the number of particles that are lost by collisional break-up, since it both decreases the collisional lifetime of disk particles (by increasing the number density of particles in the ring region) and increases the amount of time it takes for particles that are trapped in the ring to reach the inner region. Finally, if the trapping timescales, $t_{r e s}$, are longer than the age of the system, $t_{\text {sys }}$, then the trapped particles cannot have reached the inner disk yet; i.e., the ring causes a bottle-neck in the flow of particles to the inner disk. 


\section{REFERENCES}

Artymowicz, P. 1997, Annu. Rev. Earth Planet. Sci., 25, 175

Artymowicz, P., \& Clampin, M. 1997, ApJ, 490, 863

Backman, D. E., \& Paresce, F. 1993, in Protostars and Planets III, 1253

Backman, D. E., Dasgupta, A., \& Stencel, R. E. 1995, ApJ, 450, L35

Backman, D. E. 1998, in Exozodiacal Dust Workshop Conference Proceedings, eds. D. E. Backman et al. (NASA/CP-1998-10155), 13

Barrado Y Navascués, D., Stauffer, J. R., Hartmann, L., \& Balachandran, S. C. 1997, ApJ, 475, 313

Bowell, E. 1996, Asteroid Orbital Elements Database (Lowell Observatory)

Brouwer, D., \& Clemence, G. M. 1961, Methods of Celestial Mechanics (New York: Academic Press)

Burns, J. A., Lamy, P. L., \& Soter, S. 1979, Icarus, 40, 1

Cohen, M. 1999, private communication

Dermott, S. F., Nicholson, P. D., Burns, J. A., \& Houck, J. R. 1984, Nature, 312, 505

Dermott, S. F., Nicholson, P. D., Burns, J. A., \& Houck, J. R. 1985, in Properties and Interactions of Interplanetary Dust, eds. R. H. Giese, \& P. Lamy (Dordrecht: D. Reidel), 395

Dermott, S. F., \& Nicholson, P. D. 1986, Nature, 319, 115

Dermott, S. F., Gomes, R. S., Durda, D. D., Gustafson, B. A. S., Jayaraman, S., Xu, Y. L., \& Nicholson, P. D. 1992, in Chaos, Resonance and Collective Dynamical Phenomena in the Solar System, ed. S. Ferraz-Mello (IAU), 333

Dermott, S. F., Jayaraman, S., Xu, Y. L., Gustafson, B. Å. S., \& Liou, J. C. 1994, Nature, 369, 719

Dermott, S. F., Jayaraman, S., Xu, Y. L., Grogan, K., \& Gustafson, B. Å. S. 1996, in Unveiling the Cosmic Infrared Background, ed. E. Dwek (New York: AIP), 25

Dermott, S. F., Grogan, K., Holmes, E. K., \& Wyatt, M. C. 1998, in Exozodiacal Dust Workshop Conference Proceedings, eds. D. E. Backman et al. (NASA/CP-1998-10155), 59

Dermott, S. F., Grogan, K., Holmes, E. K., \& Kortenkamp, S. 1999, in Formation and Evolution of Solids in Space, ed. J. M. Greenberg (Dordrecht: Kluwer), in press

Dohnanyi, J. S. 1969, J. Geophys. Res., 74, 2531 
Draine, B. T., \& Lee, H. M. 1984, ApJ, 285, 89

Duncan, M., Quinn, T., \& Tremaine, S. 1989, Icarus, 82, 402

Durda, D. D., \& Dermott, S. F. 1997, Icarus, 130, 140

Durda, D. D., Greenberg, R., \& Jedicke, R. 1998, Icarus, 135, 431

Fajardo-Acosta, S. B., Telesco, C. M., \& Knacke, R. F. 1993, ApJ, 417, L33

Fisher, R. S., Telesco, C. M., Piña, R. K., Knacke, R. F., \& Wyatt, M. C. 1999, ApJ, submitted

Gatewood, G. 1996, BAAS, 188, 4011

Gorkavyi, N. N., Ozernoy, L. M., Mather, J. C., \& Taidakova, T. 1997, ApJ, 488, 268

Greaves, J. S., et al. 1998, ApJ, 506, L133

Grogan, K., Dermott, S. F., Jayaraman, S., \& Xu, Y. L. 1997, Planet. Space Sci., 45, 1657

Grün, E., Zook, H. A., Fechtig, H., \& Giese, R. H. 1985, Icarus, 62, 244

Gurnett, D. A., Ansher, J. A., Kurth, W. S., \& Granroth, L. J. 1997, Geo. Res. Lett., 24, 3125

Gustafson, B. Å. S. 1994, Annu. Rev. Earth Planet. Sci., 22, 553

Hirayama, K. 1918, AJ, 31, 185

Holland, W. S., et al. 1998, Nature, 392, 788

Jayaraman, S., \& Dermott, S. F. 1999, Icarus, submitted

Jayawardhana, R., Fisher, R. S., Hartmann, L., Telesco, C. M., Piña, R. K., \& Fazio, G. 1998, ApJ, 503, L79

Jewitt, D. C. 1999, AREPS, in press

Jura, M., Zuckerman, B., Becklin, E. E., \& Smith, R. C. 1993, ApJ, 418, L37

Jura, M., Ghez, A. M., White, R. J., McCarthy, D. W., Smith, R. C., \& Martin, P. G. 1995, ApJ, 445,451

Jura, M., Malkan, M., White, R., Telesco, C. M., Fisher, R. S., \& Piña, R. K. 1998, ApJ, 505, 897

Kalas, P., \& Jewitt, D. C. 1995, AJ, 110, 794

Kalas, P. 1998, Science, 281, 182

Kelsall, T., et al. 1998, ApJ, 508, 44 
Kessler, D. J. 1981, Icarus, 48, 39

Kimura, H., \& Mann, I. 1998, ApJ, 499, 454

Koerner, D. W., Ressler, M. E., Werner, M. W., \& Backman, D. E. 1998, ApJ, 503, L83

Kortenkamp, S. J., \& Dermott, S. F. 1998, Icarus, 135, 469

Laor, A., \& Draine, B. T. 1993, ApJ, 402, 441

Lecar, M., \& Franklin, F. 1997, Icarus, 129, 134

Leinert, C., \& Grün, E. 1990, in Space and Solar Physics, Vol. 20, Physics and Chemistry in Space: Physics of the Inner Heliosphere I, eds. R. Schween, \& E. Marsch (Berlin: Springer), 207

Liou, J. C. 1993, Ph.D. Thesis, Univ. of Florida

Lissauer, J. J. 1993, ARA\&A, 31, 129

Love, S. G., \& Brownlee, D. E. 1993, Science, 262, 550

Low, F. J., et al. 1984, ApJ, 278, L19

Malhotra, R. 1995, AJ, 110, 420

Malhotra, R. 1996, AJ, 111, 504

Marcy, G. W., \& Butler, R. P. 1998, ARA\&A, 36, 57

Murray, C. D., \& Dermott, S. F. 1999, Solar System Dynamics, (Cambridge: Cambridge University Press)

Reach, W. T., et al. 1995, Nature, 374, 521

Roques, F., Scholl, H., Sicardy, B., \& Smith, B. A. 1994, Icarus, 108, 37

Schneider, G., et al. 1999, ApJ, 513, L127

Sitko, M. L., Grady, C. A., Lynch, D. K., Russell, R. W., \& Hanner, M. S. 1999, ApJ, 510, 408

Stauffer, J. R., Hartmann, L. W., \& Barrado Y Navascués, B. 1995, ApJ, 454, 910

Stern, S. A. 1995, AJ, 110, 856

Stern, S. A., \& Colwell, J. E. 1997, ApJ, 490, 879

Sykes, M. V., Lebofsky, L. A., Hunten, D. M., \& Low, F. J. 1986, Science, 232, 1115

Sykes, M. V. 1990, Icarus, 84, 267 
Telesco, C. M., Decher, R., Becklin, E. E., \& Wolstencroft, R. D. 1988, Nature, 335, 51

Telesco, C. M., \& Knacke, R. F. 1991, ApJ, 372, L29

Telesco, C. M., et al. 1999, ApJ, submitted

Trilling, D. E., Benz, W., Guillot, T., Lunin, J. I., Hubbard, W. B., \& Burrows, A. 1998, ApJ, 500,428

Vedder, J. D. 1998, Icarus, 131, 283

Ward, W. R., \& Hahn, J. M. 1998, ApJ, 116, 489

Wisdom, J. 1980, AJ, 85, 1122 


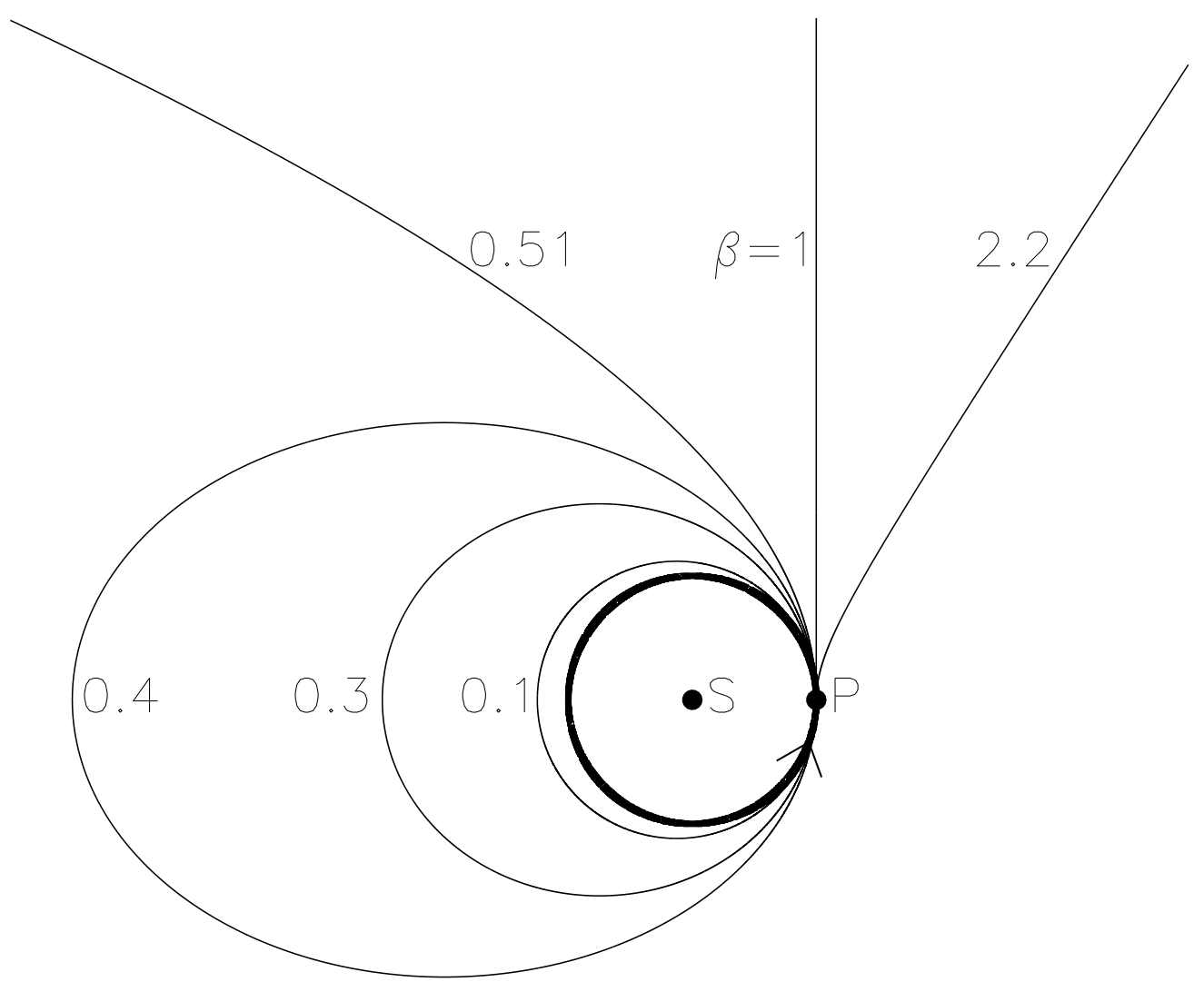

Fig. 1. - Figure showing the new orbits of the fragments of a collision in which a large parent particle "P", that was on a circular orbit around a star "S", was broken up. Fragments of different sizes have different radiation pressure forces, characterized by a particle's $\beta$, acting on them, and so have different orbits: those with $\beta<0.1$, the "large" particles, have orbits that are close to that of the parent; those with $0.1<\beta<0.5$, the " $\beta$ critical" particles, have orbits that have the same pericenter distance as the parent, but larger apocenter distances; and those with $\beta>0.5$, the " $\beta$ meteoroids", have hyperbolic orbits. Since when they are created, the velocity vector of all fragments is perpendicular to the stellar direction, this is the point of their orbit's closest approach to the star. The orbits of fragments with $\beta=0,0.1,0.3,0.4,0.51,1.0$, and 2.2 are shown here. The thick circular line denotes both the orbit of the parent particle and that of collisional fragments with $\beta=0$. All particles are orbiting the star counterclockwise. 

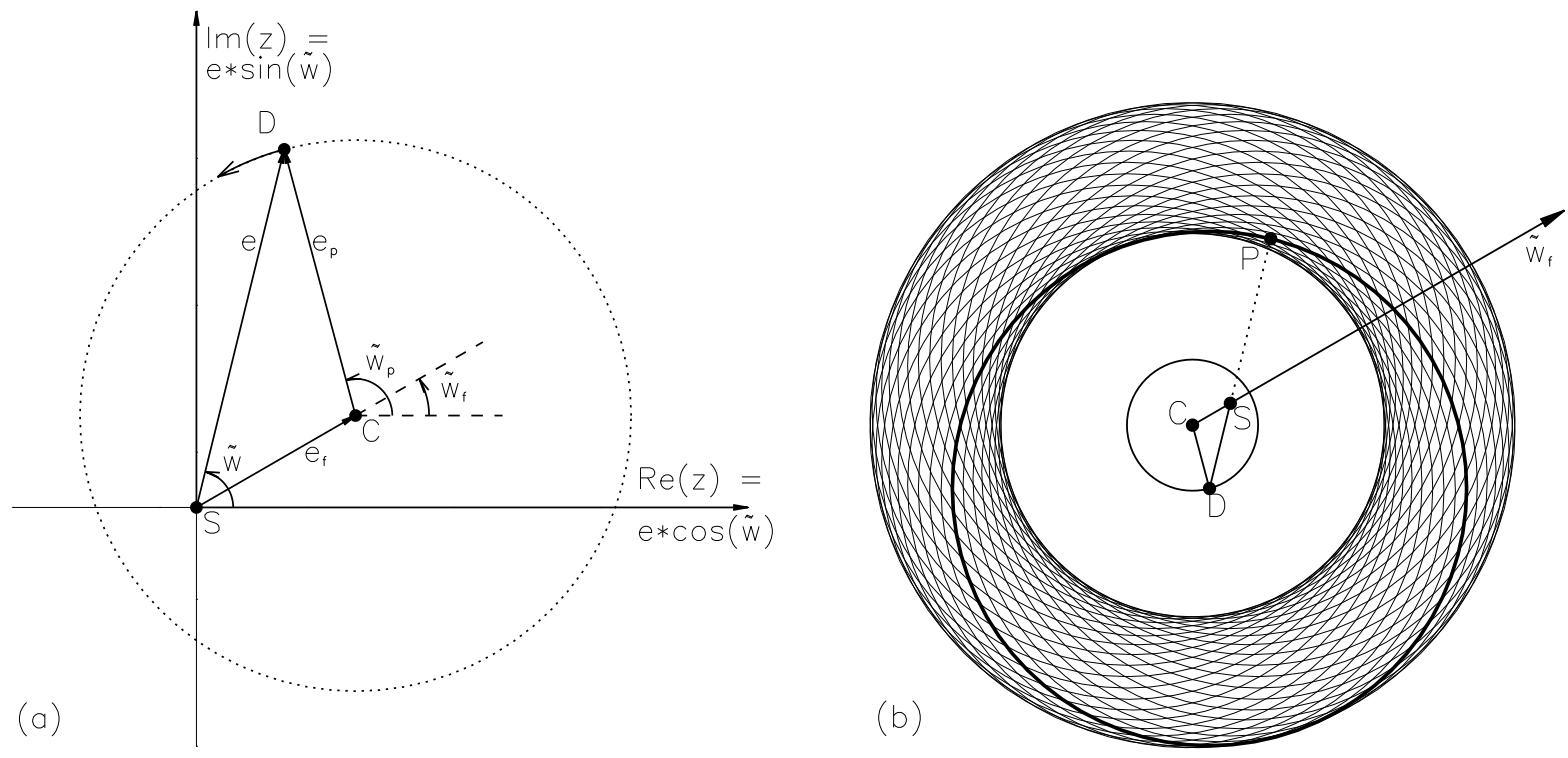

Fig. 2.- (a) The osculating (instantaneous) complex eccentricity, $z=e * \exp i \tilde{\omega}=S D$, of the orbit of a particle in a system with one or more massive perturbers can be resolved vectorially into two components: a forced eccentricity, $z_{f}=e_{f} * \exp i \tilde{\omega}_{f}=S C$, that is imposed on the particle's orbit by the perturbers; and a proper eccentricity, $z_{p}=e_{p} * \exp i \tilde{\omega}_{p}=C D$, that is the particle's intrinsic eccentricity. The secular evolution of its complex eccentricity is to precess counterclockwise around the circle in (a), although the forced eccentricity may also vary with time. Initially, the orbital elements of a family of collisional fragments created in the break-up of one large asteroid are the same as those of the original asteroid. After a few precession timescales, their complex eccentricities are evenly distributed around the circle in (a); this is because each fragment has a slightly different precession timescale. Thus, these fragments have the same $a, e_{f}, \tilde{\omega}_{f}, e_{p}$, but random $\tilde{\omega}_{p}$, and so their orbits have different eccentricities and orientations. The spatial distribution of these collisional fragments is shown in (b). Their elliptical orbits are represented here by circles of radius $a$ with centers that are offset from the star, $S$, by ae in a direction opposite to the pericenter direction, $\tilde{\omega}$. A heavy line is used to highlight the orbit of a fragment with a pericenter $P$, and displaced circle center $D$, where $D P=a$ and $S D=a e$; the triangle $S C D$, where $S C=a e_{f}$ and $C D=a e_{p}$, corresponds to a similar one in (a). Since the distribution of $\tilde{\omega}_{p}$ is random, it follows that the points $D$ for all the fragments are distributed on a circle of radius $a e_{p}$ and center $C$. Thus, the fragments form a uniform torus of inner radius $a\left(1-e_{p}\right)$, and outer radius $a\left(1+e_{p}\right)$, centered on a point $C$ displaced from the star by a distance $a e_{f}$ in a direction away from the forced pericenter, $\tilde{\omega}_{f}$ (Dermott et al. 1985). 

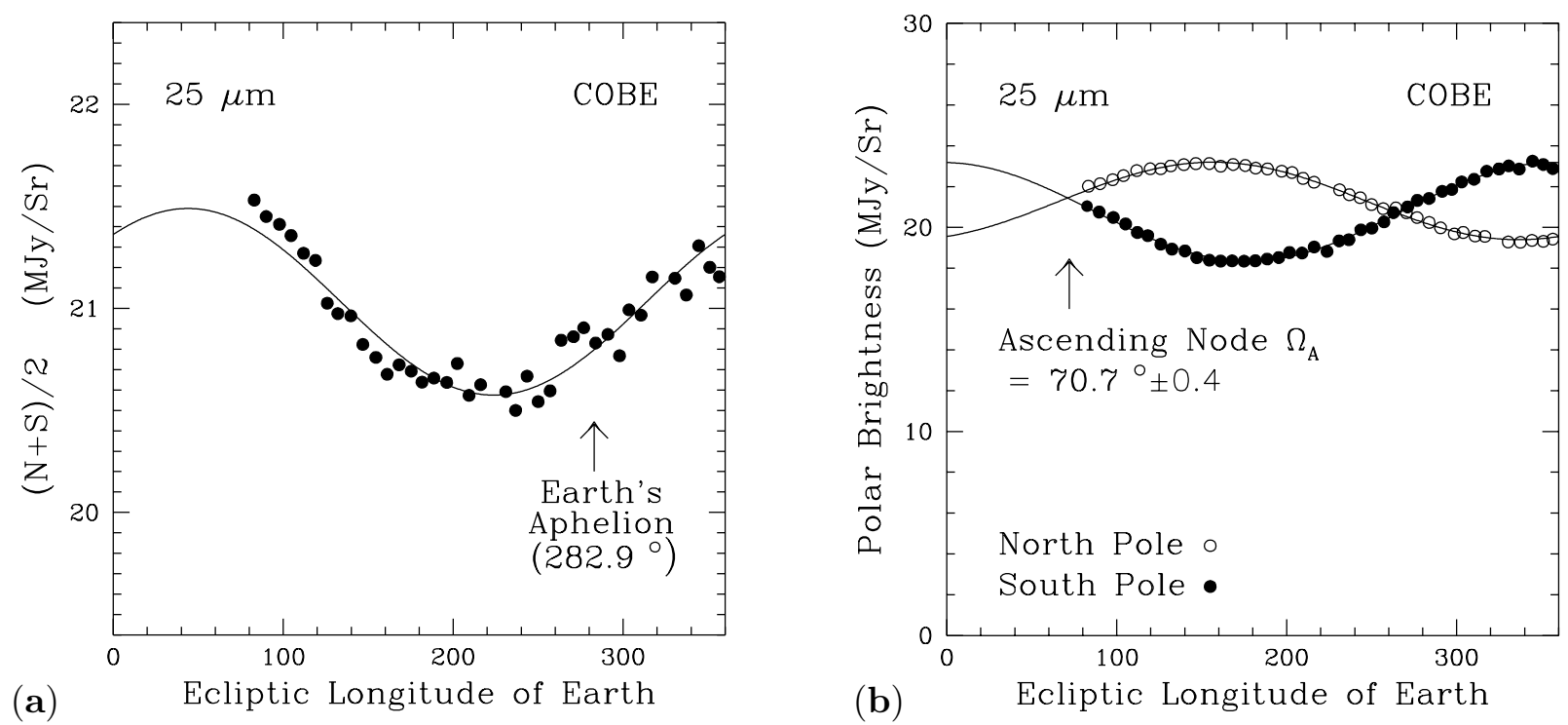

Fig. 3.- COBE observations in the $25 \mu \mathrm{m}$ waveband of the variation of brightnesses at the North, $N$, and South, $S$, ecliptic poles with ecliptic longitude of the Earth, $\lambda_{\oplus}$ (Dermott et al. 1999). (a) $N+S$ is at a minimum at $\lambda_{\oplus}=224 \pm 3^{\circ}$. The displacement of this minimum from the Earth's aphelion at $\lambda_{\oplus}=282.9^{\circ}$ implies that the center of symmetry of the zodiacal cloud is displaced from the Sun. (b) $N$ and $S$ are equal when the Earth is at either the ascending or the descending node of the plane of symmetry of the cloud at $1 \mathrm{AU}$, giving an ascending node of $70.7 \pm 0.4^{\circ}$. This plane of symmetry is different from the plane of symmetry of the cloud at distances $>1$ AU from the Sun, implying that the cloud is warped. 


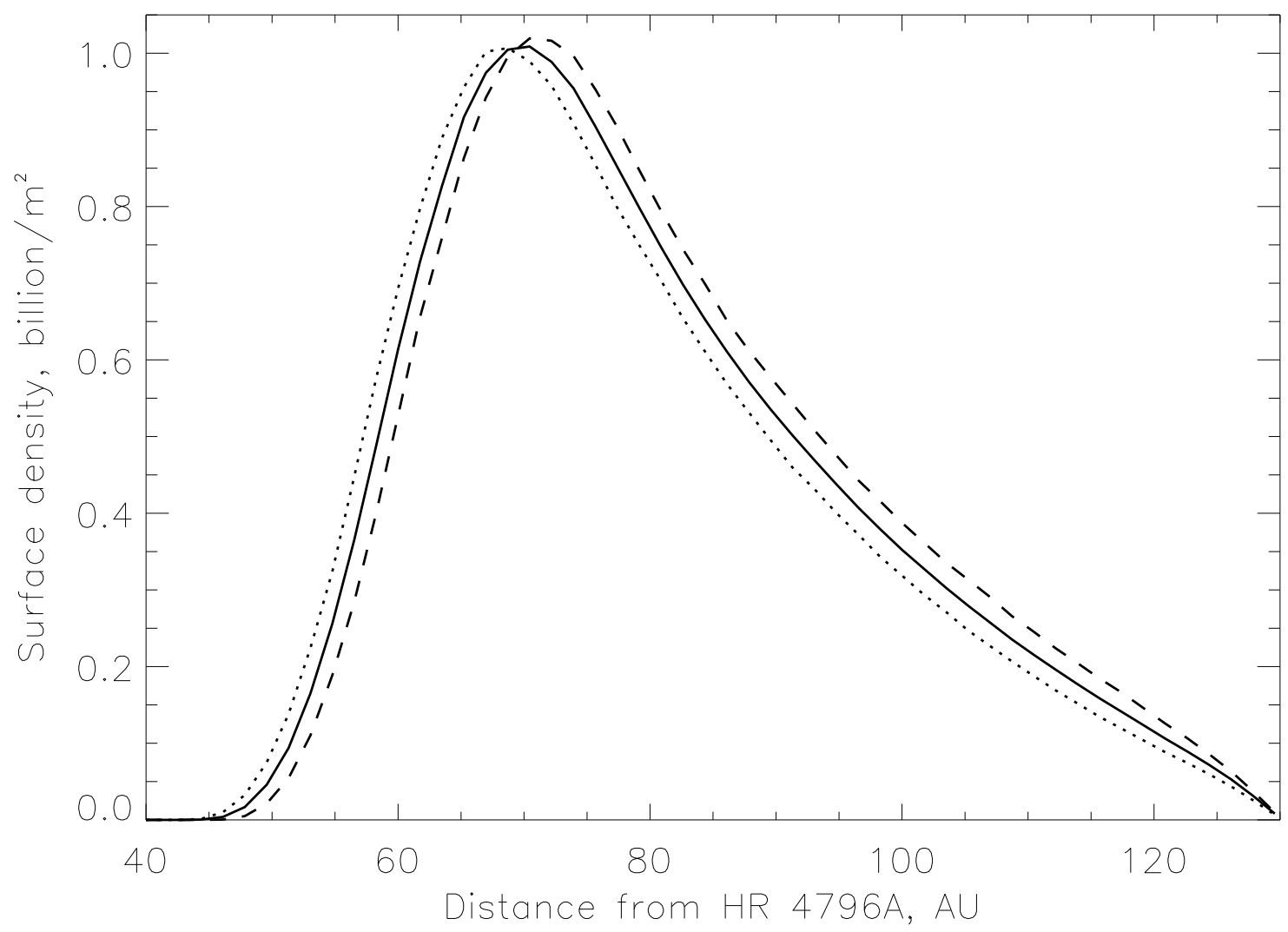

Fig. 4.- The surface number density of the $2.5 \mu \mathrm{m}$ dust grains in the HR 4796 disk derived from the $18.2 \mu \mathrm{m}$ brightness distribution (T99). The solid curve is the azimuthal average of the surface number density, and the dotted and dashed lines indicate the density through the disk towards and away from the forced pericenter direction of the model, respectively. The offset is a result of the forced eccentricity imposed on the disk model; the inner edge of each side of the disk is offset by $\sim a_{\text {min }} e_{f} \approx 1$ AU. The disk's surface density peaks at $\sim 1.02 \times 10^{9} \mathrm{~m}^{-2}$ at $\sim 70$ AU. Interior to this, the surface density falls to zero by $45 \mathrm{AU}$; the sloping cut-off is due to the eccentricities of the disk model particles' orbits. Exterior to 70 AU, the surface density falls off $\propto r^{-3}$; this is due to the distribution of the disk model particles' semimajor axes, $n(a) \propto a^{-2}$. 

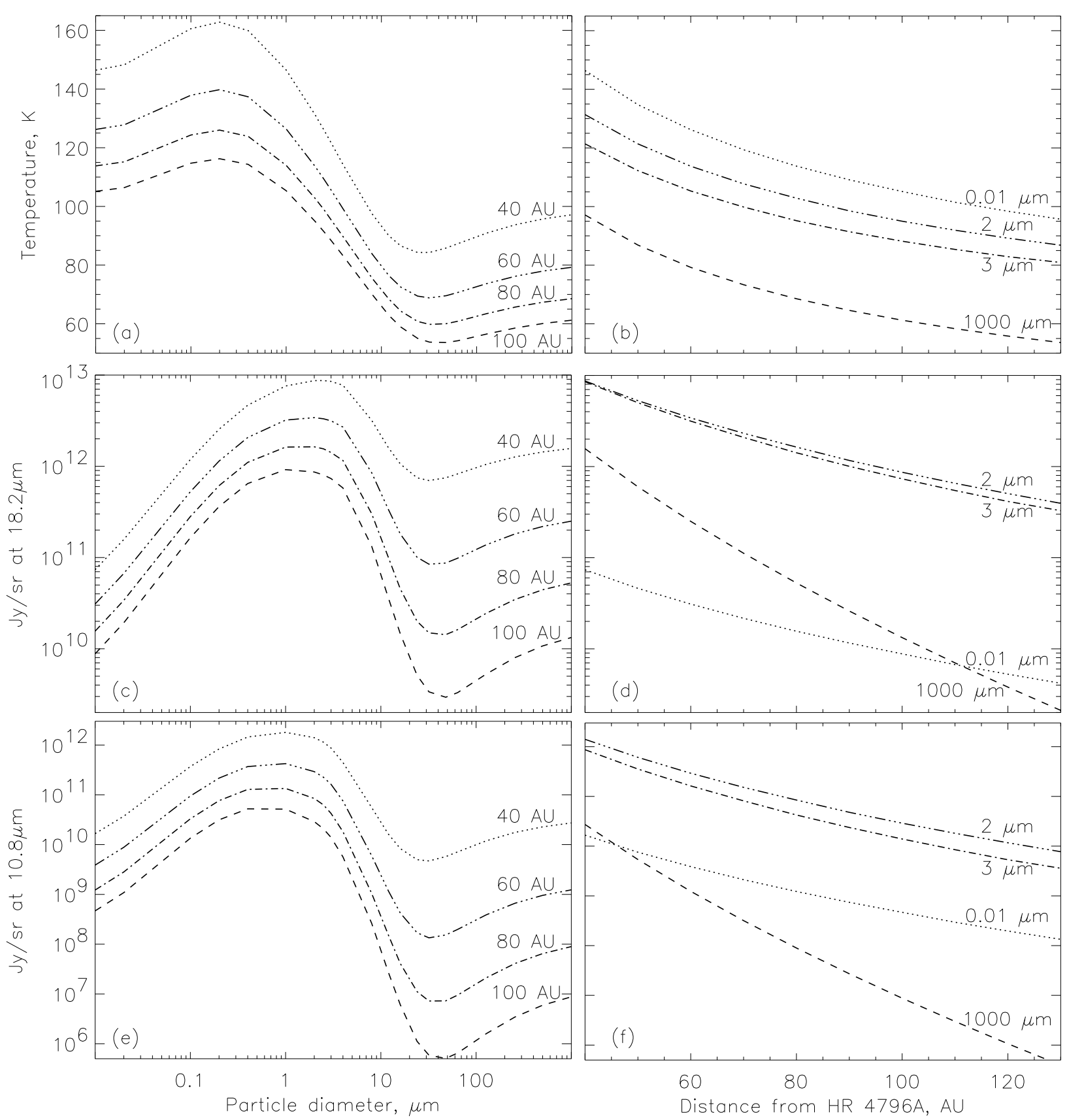

Fig. 5. - The thermal properties of astronomical silicate Mie spheres in the HR 4796 disk, plotted for particles of different sizes at 40, 60, 80, and $100 \mathrm{AU}$ from $\mathrm{HR} 4797 \mathrm{~A}$ (a), (c), and (e), and for $0.01,2,3$, and $1000 \mu \mathrm{m}$ diameter particles at different distances from HR 4796A (b), (d), and (f). The temperatures that these particles attain is plotted in (a) and (b). The contribution of a particle's thermal emission to the flux density received at the Earth per solid angle that its cross-sectional area subtends there, $Q_{a b s}(D, \lambda) B_{\nu}[T(D, r), \lambda]$ (eq. [3]), is plotted for observations in the IHW18, $18.2 \mu \mathrm{m},(\mathbf{c})$ and (d), and N, $10.8 \mu \mathrm{m},(\mathbf{e})$ and (f) wavebands. The brightnesses of disk models in these two wavebands were calculated by taking $P(\lambda, r)$ from the lines on $(\mathbf{d})$ and (f) corresponding to particles of diameter $D_{t y p}$. 


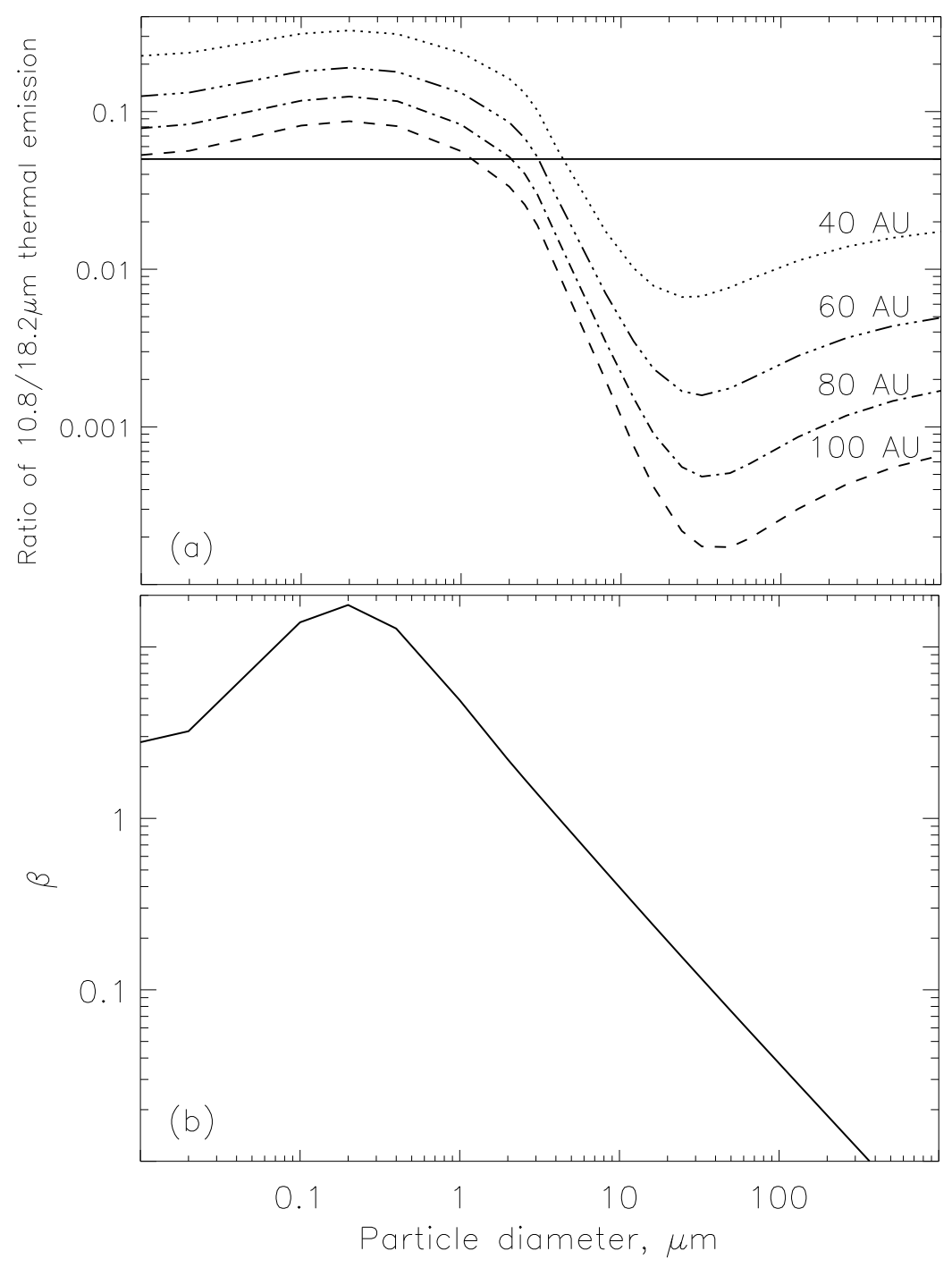

Fig. 6.- (a) The ratio of the thermal emission in the N, $10.8 \mu \mathrm{m}$, and IHW18, $18.2 \mu \mathrm{m}$, wavebands, of astronomical silicate Mie spheres of different sizes at 40, 60, 80, and 100 AU from HR 4796A

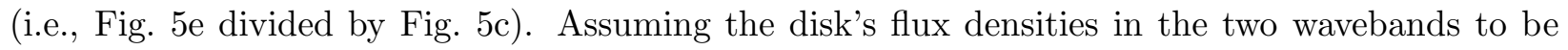
dominated by the emission of particles at 60-80 AU, the observed ratio of flux densities, $O(0.05)$ (T99; 5.2 .2 ), can be used to estimate that the disk's emitting particles have $D_{t y p}=2-3 \mu \mathrm{m}$. (b) The ratio, $\beta$, of the radiation pressure force to the gravitational force acting on astronomical silicate Mie spheres of different sizes in the HR 4796 disk. 

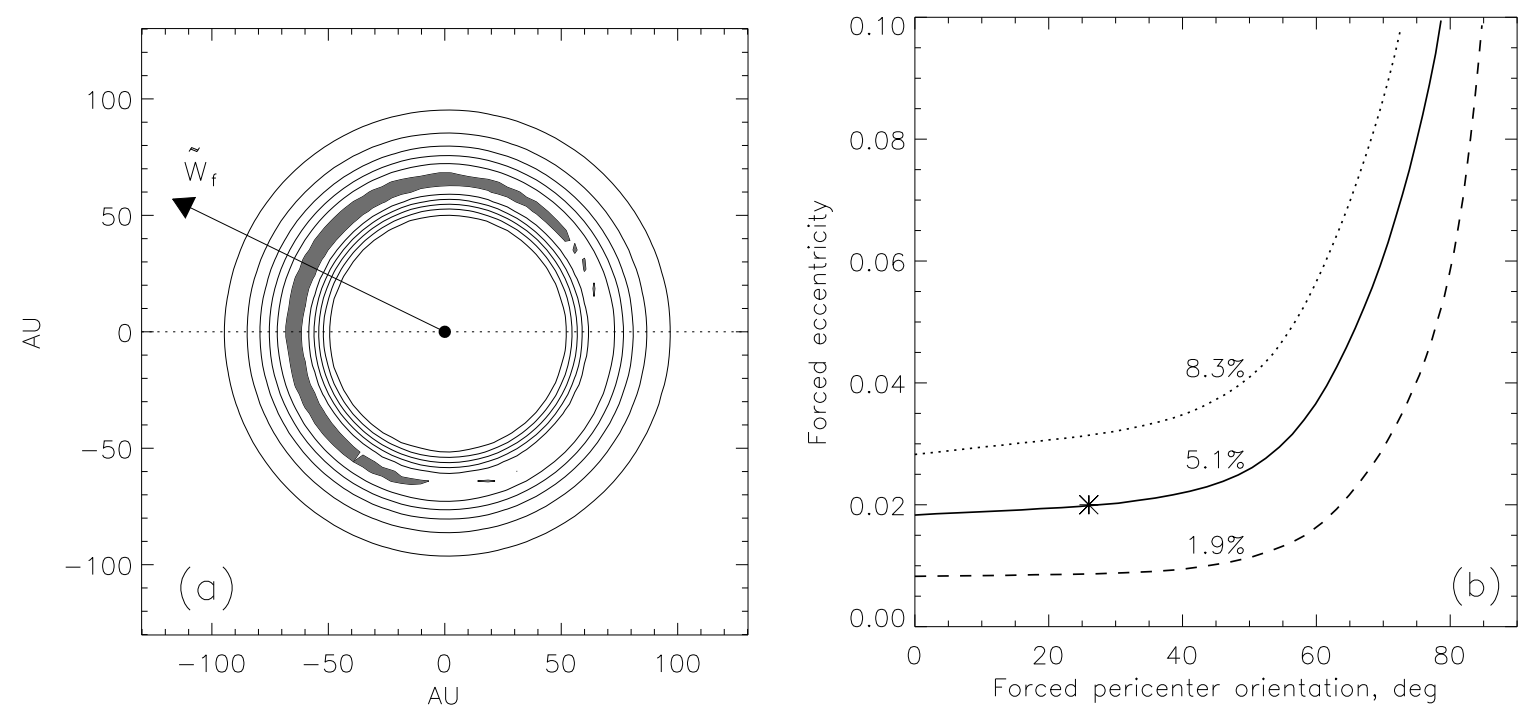

Fig. 7.- (a) Contour plot of an unsmoothed face-on view of the HR 4796 disk model (shown also in Fig. 4) seen in the IHW18 $(18.2 \mu \mathrm{m})$ waveband. The contours are spaced linearly at $0.17,0.34$, $0.51,0.68,0.85$, and $1.02 \mathrm{mJy} /$ pixel. The disk's offset causes particles in the forced pericenter direction, located at a position angle of $90^{\circ}-\tilde{\omega}_{f}$, where $\tilde{\omega}_{f}=26^{\circ}$, as measured from North in a counterclockwise direction, to be hotter, and hence brighter, than those in the forced apocenter direction; this is the "pericenter glow" phenomenon, evident in this figure by the shape of the brightest (filled-in) contour. The geometry of the observation is defined such that the disk as it is shown here is rotated by a further $90^{\circ}-I_{o b s}$ about the dotted line, where $I_{o b s}=13^{\circ}$. (b) Relation of forced eccentricity, $e_{f}$, to the orientation of the longitude of forced pericenter, $\tilde{\omega}_{f}$, in the model to achieve the observed lobe brightness asymmetry of $5.1 \pm 3.2 \%$ (a similar relationship is necessary to achieve the observed radial offset). A forced eccentricity as small as 0.02 would suffice to achieve the observed asymmetry, but a higher forced eccentricity could be necessary if the forced pericenter is aligned in an unfavorable direction. Our final model, shown in Figs. 4, 9, 9 , has $e_{f}=0.02$ and $\tilde{\omega}_{f}=26^{\circ}$, and this point is shown with an asterisk on this plot. 

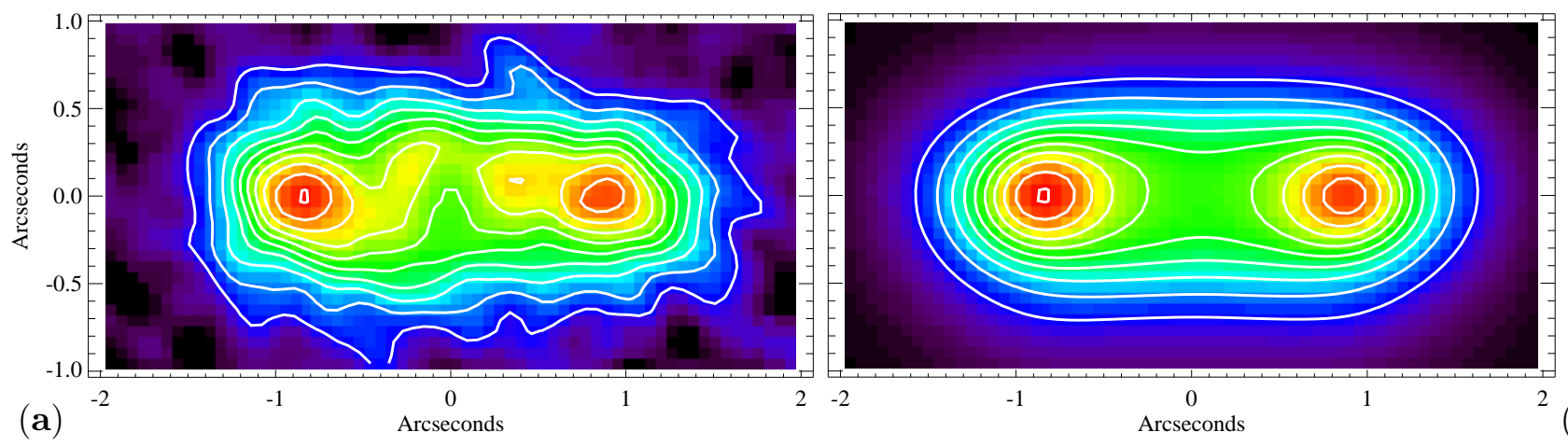

(b)
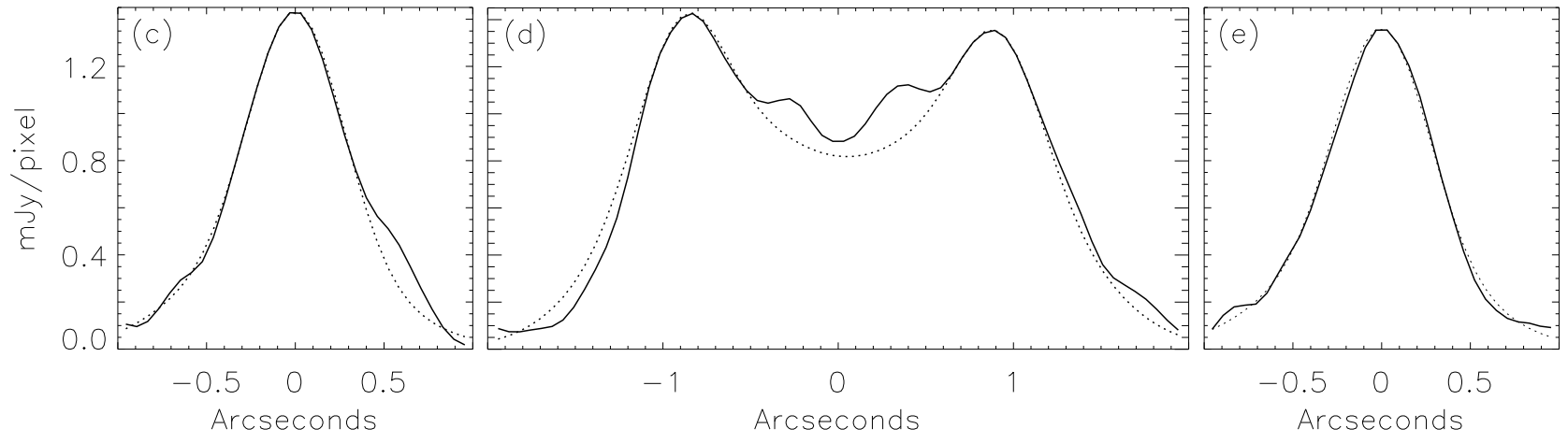

Fig. 8. - Top - False color images of HR 4796 in the IHW18 $(18.2 \mu \mathrm{m})$ waveband. Both the observation (a), on the left, and the model (b), on the right, have been rotated to horizontal with the NE lobe on the left. The contours are spaced linearly at $0.22,0.35,0.49,0.62,0.75,0.89,1.02$, $1.15,1.29$, and $1.42 \mathrm{mJy} /$ pixel. The observation has had the photospheric emission of HR 4796A subtracted, and a 3 pixel FWHM gaussian smoothing applied. The image of the model mimics the observation both in pixel size ( 1 pixel $=00^{\prime \prime} 0616=4.133$ AU) and smoothing (using an observed PSF and including the 3 pixel post-observational smoothing). Bottom - Line-cuts in the vertical direction through the NE (c) and SW (e) lobes, and in the horizontal direction through the center of both lobes (d). The observations are shown with a solid line and the model with a dotted line. 


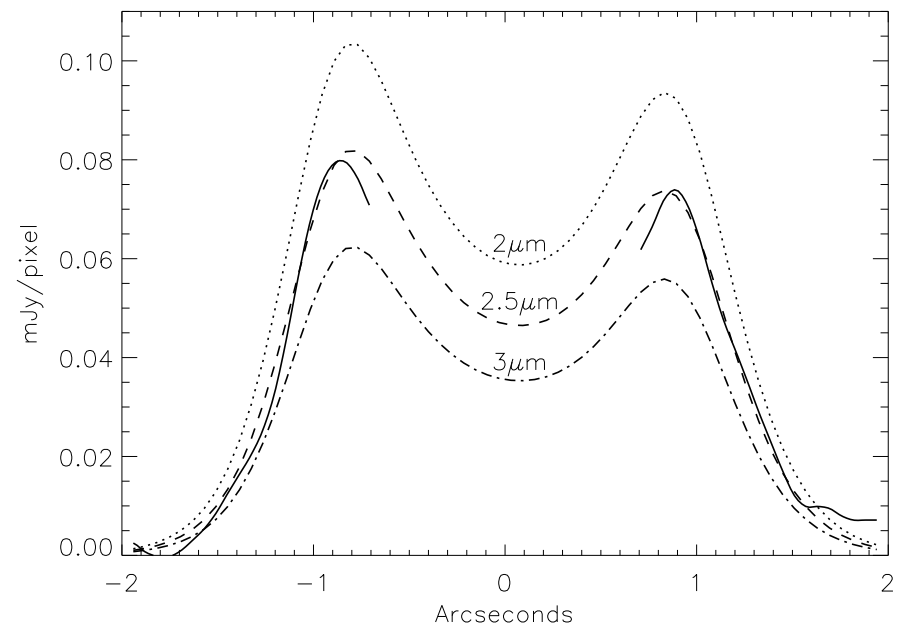

Fig. 9.- Horizontal line-cuts along the plane of the lobes in the $\mathrm{N}(10.8 \mu \mathrm{m})$ band. The observation is shown with a solid line and models with particle diameters of $D_{t y p}=2,2.5$, and $3 \mu \mathrm{m}$ are shown with dotted, dashed, and dash-dot lines. The total amount of cross-sectional area in the models, $\sigma_{t o t}$, has been scaled to fit the observed mean brightness of the lobes in the IHW18 $(18.2 \mu \mathrm{m})$ waveband; the model with $D_{t y p}=2.5 \mu \mathrm{m}$ gives the best fit to the observed lobe brightnesses in the $\mathrm{N}$ band. The observed $\mathrm{N}$ band flux density is not well constrained within 0. ". 8 of HR 4796A due to imperfect subtraction of the stellar photosphere from the image (T99), and so it is not shown here. 

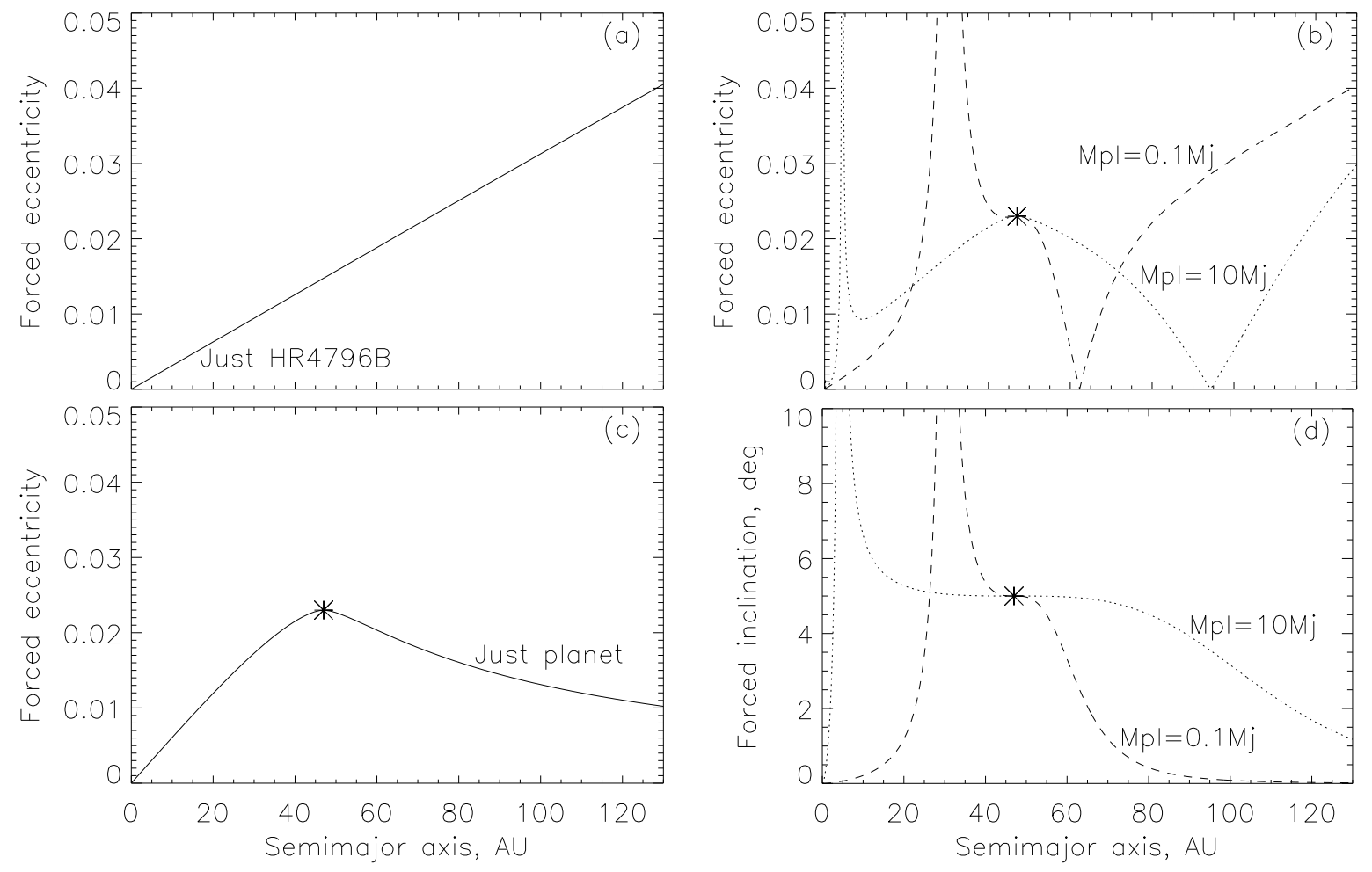

Fig. 10. - Plots of the forced eccentricities imposed on the orbits of particles in the HR 4796 system as a function of their semimajor axes, assuming different combinations of perturbers in the system: (a) HR 4796B only; (b) HR 4796B and a planet at the inner edge of the disk; (c) a planet only. The forced inclinations imposed by the two perturber system are shown in (d). The inner edge of the disk is at $a=62 \mathrm{AU}$. HR $4796 \mathrm{~B}$ and the planet are assumed to have: $M_{B}=0.38 M_{\odot}, a_{B}=517$ $\mathrm{AU}, e_{B}=0.13$, and $I_{B}=0 ; M_{p l}=0.1$ and $10 M_{J}$, where $M_{J}$ is the mass of Jupiter, $a_{p l}=47$ $\mathrm{AU}, e_{p l}=0.023$, and $I_{p l}=5^{\circ}$. The orbital elements of the planet are marked by an asterisk on the forced elements plots. In a one perturber system the forced eccentricity is independent of the mass of the perturber, and the forced inclination is the plane of the perturber's orbit. In a two perturber system, the shapes of the forced element plots depend on the mass of the planet, and the forced eccentricity also depends on the orientations of the perturbers' orbits. The forced eccentricity is plotted in (b) assuming that $\tilde{\omega}_{p l}=\tilde{\omega}_{B}+180^{\circ}$. This means that $\tilde{\omega}_{f}=\tilde{\omega}_{p l}$ for $a<a_{\text {crit }}$, and $\tilde{\omega}_{f}=\tilde{\omega}_{B}$ for $a>a_{c r i t}$, where $a_{c r i t}$ is the semimajor axis of a particle's orbit for which $e_{f}=0$; a similar alignment with the perturbers' orbital planes seen in the plot of the particles' forced inclinations. Thus, the lobes have both their asymmetries and their plane of symmetry aligned with the orbit of the planet if $M_{p l}>0.1 M_{J}$, and with the orbit of HR 4796B if $M_{p l}<0.1 M_{J}$. 